\title{
Scalar and pseudoscalar bifurcations motivated by pattern formation on the visual cortex
}

\author{
Paul C Bressloff ${ }^{1}$, Jack D Cowan ${ }^{2}$, Martin Golubitsky ${ }^{3}$ and \\ Peter J Thomas ${ }^{4}$ \\ ${ }^{1}$ Department of Mathematics, University of Utah, Salt Lake City, UT 84112-0090, USA \\ 2 Mathematics Department, University of Chicago, Chicago, IL 60637, USA \\ ${ }^{3}$ Department of Mathematics, University of Houston, Houston, TX 77204-3476, USA \\ ${ }^{4}$ Computational Neurobiology Laboratory, Salk Institute for Biological Studies, San Diego, \\ CA 92186-5800, USA
}

Received 10 August 2000, in final form 1 March 2001

Recommended by F Otto

\begin{abstract}
Bosch Vivancos, Chossat and Melbourne showed that two types of steadystate bifurcations are possible from trivial states when Euclidean equivariant systems are restricted to a planar lattice—scalar and pseudoscalar—and began the study of pseudoscalar bifurcations. The scalar bifurcations have been well studied since they appear in planar reaction-diffusion systems and in plane layer convection problems. Bressloff, Cowan, Golubitsky, Thomas and Wiener showed that bifurcations in models of the visual cortex naturally contain both scalar and pseudoscalar bifurcations, due to a different action of the Euclidean group in that application.

In this paper, we review the symmetry discussion in Bressloff et al and we continue the study of pseudoscalar bifurcations. Our analysis furthers the study of pseudoscalar bifurcations in three ways.

(a) We complete the classification of axial subgroups on the hexagonal lattice in the shortest wavevector case proving the existence of one new planform-a solution with triangular $D_{3}$ symmetry.

(b) We derive bifurcation diagrams for generic bifurcations giving, in particular, the stability of solutions to perturbations in the hexagonal lattice. For the simplest (codimension zero) bifurcations, these bifurcation diagrams are identical to those derived by Golubitsky, Swift and Knobloch in the case of Bénard convection when there is a midplane reflectionthough the details in the analysis are more complicated.

(c) We discuss the types of secondary states that can appear in codimensionone bifurcations (one parameter in addition to the bifurcation parameter), which include time periodic states from roll and hexagon solutions and drifting solutions from triangles (though the drifting solutions are always unstable near codimension-one bifurcations). The essential difference between scalar and pseudoscalar bifurcations appears in this discussion.
\end{abstract}

Mathematics Subject Classification: 37G40, 58E09, 92C20 


\section{Introduction}

Bifurcations to doubly periodic solutions in planar systems of Euclidean invariant partial differential equations have been well studied in a number of contexts. Standard examples include reaction-diffusion systems, the Boussinesq equations modelling convection (both with and without midplane symmetry and with and without rotation), and two-dimensional NavierStokes equations. Typically, these analyses are used to prove the existence of regular patterned solutions or planforms. The types of patterns that occur in these examples and their stability are different and the cause of these differences is symmetry.

Ermentrout and Cowan [6] showed that a bifurcation analysis based on finding doubly periodic solutions can be used when studying pattern formation in the visual cortex, where the patterns can be interpreted as visual hallucination patterns. More recently, Bressloff et al [2] have shown that when additional structure in the visual cortex is taken into account in the models, the symmetry properties of the equations change and, hence, the bifurcation analysis and the type of patterns are again different. In this paper we provide the mathematical description of the bifurcation analysis used in [2].

There is a common approach to all lattice bifurcation problems, which we now describe. Let $\lambda$ be a bifurcation parameter and assume that the equations have a spatially homogeneous solution for all $\lambda$.

(a) A linear analysis of the homogeneous solution leads to a dispersion curve. More precisely, translation symmetry implies that eigenfunctions have a plane wave $w_{k}(\boldsymbol{x})=\mathrm{e}^{2 \pi \mathrm{i} k \cdot \boldsymbol{x}}$ factor where $k \in \mathbb{R}^{2}$. Rotation symmetry implies that the linearized equations have infinite-dimensional eigenspaces, that is, instability occurs simultaneously to all functions $w_{k}(\boldsymbol{x})$ with constant $k=|\boldsymbol{k}|$. The number $k$ is called the wavenumber. Points $(k, \lambda)$ on the dispersion curve are the minimum values of $\lambda$ for which an instability of the homogeneous solution to an eigenfunction with wavenumber $k$ occurs.

(b) Often, in these examples, the dispersion curve has a unique minimum, that is, there is a critical wavenumber $k_{*}$ at which the first instability of the homogeneous solution occurs. Bifurcation analyses near such points are difficult for two reasons: the kernel of the linearization is infinite dimensional and there is a continuum of zero eigenvalues as $k$ is varied (as demonstrated by the dispersion curve).

(c) These difficulties can be side-stepped by considering a restricted class of possible solutions: a planar lattice $\mathcal{L}$ is chosen and the differential operator is restricted to the space $\mathcal{F}_{\mathcal{L}}$ of functions that are doubly periodic with respect to $\mathcal{L}$.

(d) The symmetries of the restricted problem change from Euclidean symmetry in two ways. First, only a finite number of rotations and reflections remain as symmetries. Let the holohedry $H_{\mathcal{L}}$ be the group of rotations and reflections that preserve the lattice $\left(\boldsymbol{D}_{2}\right.$ for rhombic lattices, $\boldsymbol{D}_{4}$ for square lattices and $\boldsymbol{D}_{6}$ for hexagonal lattices). Second, translations act on the restricted problem modulo $\mathcal{L}$; that is, translations act as a torus $T^{2}$. Therefore, the symmetry group of the lattice problem is (the compact group) $\Gamma_{\mathcal{L}}=H_{\mathcal{L}}+T^{2}$.

(e) It follows from double periodicity that the kernel of the restricted problem is typically finite dimensional, since only those rotations in $H_{\mathcal{L}}$ are symmetries of the restricted problem, and they are finite in number.

(f) The restricted bifurcation problem must be further specialized. First, a lattice type needs to be chosen (rhombic, square and hexagonal). Second, the size of the lattice must be chosen so that a plane wave with critical wavenumber $k_{*}$ is an eigenfunction in the space $\mathcal{F}_{\mathcal{L}}$. 
(g) Those $k$ for which the plane wave $\mathrm{e}^{2 \pi \mathrm{i} k \cdot x}$ is doubly periodic with respect to $\mathcal{L}$ (that is, the plane wave is in $\mathcal{F}_{\mathcal{L}}$ ) are called dual wavevectors. The set of dual wavevectors is a lattice, called the dual lattice, and is denoted by $\mathcal{L}^{*}$. In this paper we consider only those lattice sizes where the critical dual wavevector are vectors of shortest length in $\mathcal{L}^{*}$. Generically, there is just one eigenfunction of the linearized operator $L$ restricted to $\mathcal{F}_{\mathcal{L}}$ corresponding to each shortest length dual wavevector. That is, we expect $\operatorname{ker} L=\mathbb{R}^{n}$ where $n$ is four, four, and six on the rhombic, square and hexagonal lattices, respectively.

(h) Since ker $L$ is finite dimensional, we can use Lyapunov-Schmidt or centre manifold reduction to obtain implicitly a system of reduced bifurcation equations on $\mathbb{R}^{n}$ whose zeros are in one-to-one correspondence with the steady states of the original equation. Moreover, this reduction can be performed so that the reduced bifurcation equations are $\Gamma_{\mathcal{L}}$-equivariant.

(i) Solving the reduced bifurcation equations is still a difficult problem. This problem can be simplified as follows. A subgroup $\Sigma \subset \Gamma_{\mathcal{L}}$ is axial if $\operatorname{dim} \operatorname{Fix}(\Sigma)=1$ where

$$
\operatorname{Fix}(\Sigma)=\{x \in \operatorname{ker} \mathrm{L}: \sigma x=x, \forall \sigma \in \Sigma\} .
$$

The equivariant branching lemma states that generically there exists a branch of solutions corresponding to each axial subgroup. These solution types are then classified by finding all axial subgroups. (To avoid redundant solution types this classification is only made up to conjugacy and $\Sigma$ is assumed to be an isotropy subgroup.)

(j) The linearized stability of solutions obtained using the equivariant branching lemma is not straightforward to determine. The stability question has several levels: stability to all perturbations; stability to spatially doubly periodic perturbations of any lattice; and stability to perturbations or the specific lattice on which the solution is found. The last statement of stability is the easiest to carry out (it is still complicated, but representation theory helps) and it is only this type of stability that we consider in this paper.

The classification of axial subgroups and their stabilities to lattice perturbations for Euclidean group actions associated with reaction-diffusion equations and convection equations has been repeated in a variety of contexts. For example, on the hexagonal lattice, this classification leads to rolls and hexagons for reaction-diffusion equations and convection equations without the midplane reflection $[4,5]$ and to rolls, hexagons, triangles and rectangles in convection equations with the midplane reflection [9]. Bosch Vivancos et al [1] showed that different actions of the Euclidean group $\boldsymbol{E}(2)$ lead to different types of solutions. In particular, they called the standard action of $\boldsymbol{E}(2)$ that appears in reaction-diffusion equations the scalar action. They then showed the existence of another action of $\boldsymbol{E}(2)$ on functions on $\mathbb{R}^{2}$, called the pseudoscalar action. This action occurs, for example, in the equations for the stream function in the 2D Navier-Stokes equations and leads to solutions with symmetry groups different from those that appear in the scalar action.

In this paper we study bifurcation of planforms for equations measuring activity levels in the visual cortex. These equations possess a different action of the Euclidean group (on $\mathbb{R}^{2} \times S^{1}$ rather than on $\mathbb{R}^{2}$ ) - one that leads naturally to both the scalar and the pseudoscalar actions of $\boldsymbol{E}(2)$ in one problem. We analyse these bifurcations, showing that on the hexagonal lattice there are four types of axial subgroups (with symmetries similar but not identical to those found in the Boussinesq equations with the midplane reflection). We shall also discuss when these solutions are asymptotically stable (to perturbations by functions in $\mathcal{F}_{\mathcal{L}}$ ).

Our analysis furthers the study of pseudoscalar bifurcations in three ways.

(a) We complete the classification of axial subgroups on the hexagonal lattice in the shortest wavevector case proving the existence of one new planform-a solution with triangular $D_{3}$ symmetry. 
(b) We derive bifurcation diagrams for generic bifurcations giving, in particular, the stability of solutions to perturbations in the hexagonal lattice. For the simplest (codimension-zero) bifurcations, these bifurcation diagrams are identical to those derived in [9] in the case of Bénard convection when there is a midplane reflection —-though the details in the analysis are more complicated.

(c) We discuss the types of secondary states that can appear in codimension-one bifurcations (one parameter in addition to the bifurcation parameter), which includes time-periodic states from roll and hexagon solutions and drifting solutions from triangles (though the drifting solutions are always unstable near codimension-one bifurcations). The essential difference between scalar and pseudoscalar bifurcations appears in this discussion.

Finally, in order to facilitate pattern formation studies in the visual cortex, we summarize all of the lattice bifurcations in notation adapted to visual cortex models. The visual cortex models studied in [2] provide the first example of pseudoscalar bifurcations that are relevant in applications.

\section{An $E(2)$ action motivated by the visual cortex}

Neurons in the primary visual cortex V1 are known to be sensitive to the orientation of contours in the visual field. As discussed in [2], the pattern of interconnection of these neurons has interesting symmetry properties and these symmetries seem to be responsible in part for the types of geometric patterns that are reported in visual hallucinations. Using microelectrodes, voltage sensitive dyes and optical imaging, scientists have accumulated much information about the distribution of orientation selective cells in V1 and about their pattern of interconnection. These studies can be interpreted to suggest that approximately every millimetre there is an iso-orientation patch of a given orientation preference and that a set of orientation patches covering the orientation domain $[0, \pi)$ (for each eye) occurs (in humans) in a millimetre square slab of V1. This slab was called a hypercolumn by Hubel and Wiesel [10].

Thus, there seem to be at least two length scales:

(a) local: cells less than a millimetre apart tend to make connections with most of their neighbours in a roughly isotropic fashion; and

(b) lateral: cells make contacts only every millimetre or so along their axons with cells in similar iso-orientation patches.

The experimental description of the local and lateral connections in V1 is illustrated in figure 1 . The neurons in each hypercolumn are all-to-all coupled, while the connections between hypercolumns couple only those neurons that are sensitive to the same contour orientation. Moreover, if two hypercolumns lie in a direction $\phi$ from each other in V1, then only those neurons sensitive to contours oriented at angle $\phi$ are connected. Except for boundaries these connections are the same at every hypercolumn in V1.

The lateral connections are important because they change the way symmetries act. Observe that if the whole visual cortex is rotated so that hypercolumns are rotated to hypercolumns, then the lateral connections will no longer be correct-the lateral connections will not couple neurons sensitive to the direction of that connection. (The pattern of lateral connections is one way in which directions in the outside world are encoded in the visual cortex.) Note, however, that rotation symmetry can be recovered if the cells in each hypercolumn are permuted to compensate for the rotation. This shift-twist symmetry of rotation appears in (2.1). 


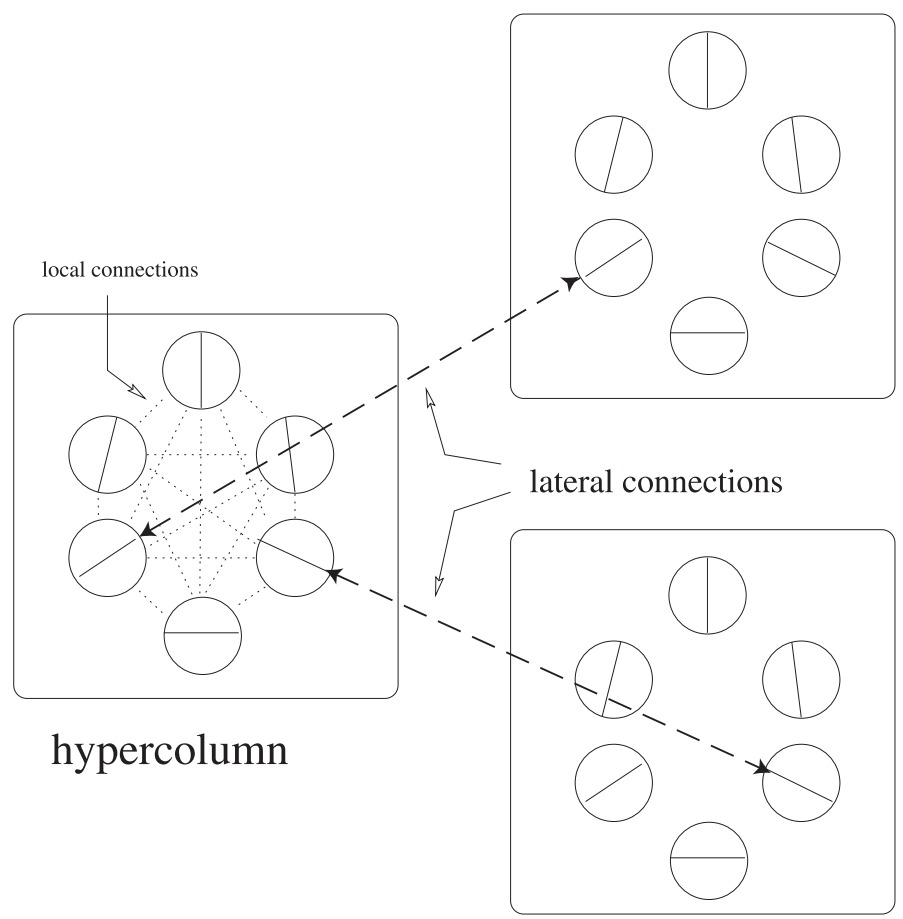

Figure 1. Illustration of isotropic local and anisotropic lateral connection patterns.

The pattern of connections illustrated in figure 1 suggests that a continuum model of the visual cortex is defined on the space $\mathbb{R}^{2} \times \boldsymbol{S}^{1}$. That is, each hypercolumn is idealized to a circle—one for each point in the plane $\mathbb{R}^{2}$ (at least when boundaries are ignored). Moreover, the action of the Euclidean group on $\mathbb{R}^{2} \times S^{1}$ is generated by

$$
\begin{array}{ll}
\boldsymbol{y} \cdot(\boldsymbol{x}, \phi)=(\boldsymbol{x}+\boldsymbol{y}, \phi) & \boldsymbol{y} \in \mathbb{R}^{2} \\
\theta \cdot(\boldsymbol{x}, \phi)=\left(R_{\theta} \boldsymbol{x}, \phi+\theta\right) & \theta \in \boldsymbol{S}^{1} \\
\boldsymbol{\kappa} \cdot(\boldsymbol{x}, \phi)=(\kappa \boldsymbol{x},-\phi) &
\end{array}
$$

where $\kappa$ is the reflection $\left(x_{1}, x_{2}\right) \mapsto\left(x_{1},-x_{2}\right)$ and $R_{\theta}$ is the rotation

$$
R_{\theta}\left(\begin{array}{c}
x \\
y
\end{array}\right)=\left(\begin{array}{cc}
\cos \theta & -\sin \theta \\
\sin \theta & \cos \theta
\end{array}\right)\left(\begin{array}{l}
x \\
y
\end{array}\right) \text {. }
$$

Finally, any state modelling the visual cortex has the form $a(x, \phi)$, where

$$
a(\boldsymbol{x}, \phi+\pi)=a(\boldsymbol{x}, \phi)
$$

since contours are not oriented. The action of $\gamma \in \boldsymbol{E}(2)$ on the function $a$ is given by

$$
\gamma a(\boldsymbol{x}, \phi)=a\left(\gamma^{-1}(\boldsymbol{x}, \phi)\right) .
$$

For example, $\theta \in \boldsymbol{S O}(2)$ acts by

$$
\left.\theta a(\boldsymbol{x}, \phi)=a\left(R_{-\theta} \boldsymbol{x}, \phi-\theta\right)\right) .
$$

Visual hallucinations are seen both by blind subjects and in sealed dark rooms, thus suggesting that they are generated in the brain and not in the eyes. Following Ermentrout and 
Cowan [6], we assume that geometric visual hallucinations are generated ab initio mostly in V1. That is, we presume that drugs act uniformly on activity levels in the visual cortex, thus causing symmetry-breaking bifurcations to patterns on the visual cortex. In [2] we show that the patterns that are formed through these bifurcations are (after a natural reinterpretation in retinal coordinates) remarkably similar to those patterns recorded by those who have experienced visual hallucinations. Since the patterns that form are directly related to the presumed coupling architecture of V1 and the symmetries it generates, this analysis supports the abstracted view of V1 described previously.

Bressloff et al [2] show that both scalar and pseudoscalar bifurcations occur in reasonable visual cortex models (based on variants of the Wilson-Cowan equations [13] that include lateral coupling and respect Euclidean symmetry) and hence that the bifurcations associated with both representations need to be analysed. In the remainder of this paper we present the details of the bifurcation analysis on doubly periodic domains described in section 1 using the state space and Euclidean group action given in (2.1).

\section{The actions of $\Gamma_{\mathcal{L}}$ on kernels of linearizations}

In $(\mathrm{g})$ in section 1 we stated that we would only consider bifurcations on lattices corresponding to dual wavevectors of the shortest length and we use that assumption here when discussing the irreducible subspaces of $\Gamma_{\mathcal{L}}$. Without loss of generality we may assume that the shortest length is 1 ; it follows (after a rotation of the lattice, if necessary) that $k=(1,0)$ is one of these shortest-length dual wavevectors.

\section{The irreducible subspaces}

For each $\boldsymbol{k} \in \mathcal{L}^{*}$ define the infinite-dimensional subspaces

$$
W_{k}=\left\{u(\phi) \mathrm{e}^{2 \pi \mathrm{i} k \cdot x}+\text { c.c. }: u: S^{1} \rightarrow \mathbb{C} \text { is } \pi \text {-periodic }\right\} .
$$

Note that $W_{-k}=W_{k}$ and that $\gamma W_{k}=W_{\gamma k}$ for any $\gamma \in \boldsymbol{O}(2)$. (Each subspace $W_{k}$ is an isotypic component for an irreducible representation of $\boldsymbol{T}^{2} ; W_{k}$ is the sum of all $\boldsymbol{T}^{2}$-irreducible subspaces where the action of $\gamma \in T^{2}$ is isomorphic to $\gamma z=\mathrm{e}^{2 \pi \mathrm{i} k \cdot x} z$. It follows that any eigenfunction of a linear operator commuting with $\boldsymbol{E}(2)$ must lie in $W_{k}$ for some wavevector k.)

Now fix $k=(1,0)$. The space

$$
W=\sum_{\gamma \in H_{\mathcal{L}}} W_{\gamma k}
$$

consists of all functions in $\mathcal{F}_{\mathcal{L}}$ corresponding to wavevectors of shortest length.

We claim that the subspace $W_{k}$ splits into two $\Gamma_{\mathcal{L}}$-invariant subspaces because

$$
\kappa u(\phi) \mathrm{e}^{2 \pi \mathrm{i} k \cdot x}=u(-\phi) \mathrm{e}^{2 \pi \mathrm{i} k \cdot x} .
$$

It follows that

$$
\begin{aligned}
& W_{k}^{+}=\left\{u(\phi) \mathrm{e}^{2 \pi \mathrm{i} k \cdot x}+\text { c.c. } \in W_{k}: u(-\phi)=u(\phi) \in \mathbb{C}\right\} \\
& W_{k}^{-}=\left\{u(\phi) \mathrm{e}^{2 \pi \mathrm{i} k \cdot x}+\text { c.c. } \in W_{k}: u(-\phi)=-u(\phi) \in \mathbb{C}\right\}
\end{aligned}
$$

are $\kappa$-invariant subspaces and $W_{k}=W_{k}^{+} \oplus W_{k}^{-}$. We call functions in $W_{k}^{+}$even and functions in $W_{k}^{-}$odd. 
Fix $u(\phi) \mathrm{e}^{2 \pi \mathrm{i} k \cdot x}+$ c.c. $\in W_{k}$, where $u(\phi)$ is real-valued and let $Z_{u}$ be the two-dimensional real subspace

$$
Z_{u}=\left\{z u(\phi) \mathrm{e}^{2 \pi \mathrm{i} k \cdot x}+\text { c.c. }: z \in \mathbb{C}\right\} .
$$

Note that $Z_{u}$ is $\boldsymbol{T}^{2}$-irreducible and that

$$
Z(u)=\sum_{\gamma \in H_{\mathcal{L}}} \gamma Z_{u}
$$

is $\Gamma_{\mathcal{L}}$-invariant.

Lemma 3.1. The subspace $Z(u)$, where $u$ is real-valued and $u(\phi) \mathrm{e}^{2 \pi \mathrm{i} k \cdot x}+$ c.c. is either even or odd, is $\Gamma_{\mathcal{L}}$ absolutely irreducible. Moreover, these subspaces are all of the $\Gamma_{\mathcal{L}}$-irreducible subspaces of $W$.

Proof. We begin by showing that every $\Gamma_{\mathcal{L}^{-}}$-irreducible subspace $Z \subset W$ has the form $Z(u)$ for some real-valued $u$ that is either odd or even. Using $T^{2}$-invariance $Z$ must contain a non-zero function in $W_{\gamma k}$ for some $\gamma \in H_{\mathcal{L}}$. After applying $\gamma^{-1}, Z$ contains a non-zero function of the form

$$
u(\phi) \mathrm{e}^{2 \pi \mathrm{i} k \cdot x}+\text { c.c. }
$$

Rotating (3.1) by $R_{\pi}$ and using the fact that $u(\phi)$ is $\pi$-periodic shows that

$$
\overline{u(\phi)} \mathrm{e}^{2 \pi \mathrm{i} k \cdot x}+\text { c.c. }
$$

is also in $Z$. Since one of $\operatorname{Re}(u)$ and $\operatorname{Im}(u)$ is non-zero, we can use linear combinations and multiplication by $\mathrm{i}$ (which is a translation symmetry) to see that (3.1) is in $Z$ where $u$ is nonzero and real-valued. Next, write $u=u^{+}+u^{-}$, where $u^{+}$is even and $u^{-}$is odd. Applying $\kappa$ shows that

$$
\left(u^{+}-u^{-}\right) \mathrm{e}^{2 \pi \mathrm{i} k \cdot x}+\text { c.c. }
$$

is in $Z$. Since one of $u^{+}$and $u^{-}$is non-zero, we can use linear combinations to see that (3.1) is in $Z$ where $u$ is non-zero, real-valued and either even or odd. It follows that $Z(u) \subset Z$ is non-zero and $\Gamma_{\mathcal{L}}$-invariant. Therefore, $Z=Z(u)$.

Next we show that $Z(u)$ is $\Gamma_{\mathcal{L}}$ absolutely irreducible. We must show that the only linear mappings $M$ on $Z(u)$ that commute with the action of $\Gamma_{\mathcal{L}}$ are scalar multiples of the identity. Choose elements $\gamma_{j} \in H_{\mathcal{L}}$ so that

$$
Z(u)=\gamma_{1} Z_{u} \oplus \cdots \oplus \gamma_{s} Z_{u}
$$

where $\gamma_{1}=I d$. Because $\gamma_{j} Z_{u}$ are isotypic components for the $T^{2}$ action, $M\left(\gamma_{j} Z_{u}\right) \subset \gamma_{j} Z_{u}$. Since $\boldsymbol{T}^{2}$ acts as $\boldsymbol{S O}(2)$ on the two-dimensional subspace $\gamma_{j} Z_{u}, M \mid \gamma_{j} Z_{u}$ is a scalar multiple of a rotation matrix. Since $R_{\pi}$ acts as a reflection on $\gamma_{j} Z_{u}$ (it is complex conjugation in complex coordinates), $M \mid \gamma_{j} Z_{u}$ is a scalar multiple of the identity. Because $H_{\mathcal{L}}$ acts transitively on the components of $Z(u)$ that scalar must be the same for all components. Thus, $M$ is a scalar multiple of the identity. 


\section{Centre subspaces on lattices}

Suppose that we consider a nonlinear differential equation whose associated differential operator $N$ has a domain consisting of functions $U: \mathbb{R}^{2} \times S^{1} \rightarrow \mathbb{R}$. Suppose also that $N$ is equivariant with respect to the action of the Euclidean group in (2.1) and that $\mathrm{N}(0)=0$. Let $\mathrm{L}$ be the linearization of $\mathrm{N}$ at 0 . Then $\mathrm{L}$ is also Euclidean equivariant. We consider parameter values where a bifurcation from $U=0$ occurs; that is, parameter values where $L$ has a zero eigenvalue. Moreover, we consider bifurcations on lattices $\mathcal{L}$ where the eigenfunctions correspond to dual wavevectors of shortest length.

Since ker $L$ is $\Gamma_{\mathcal{L}}$-invariant, it contains a $\Gamma_{\mathcal{L}}$-irreducible subspace $Z(u)$; moreover, generically, ker $L$ is irreducible. It follows that two types of bifurcation can occur on lattices with the symmetries (2.1) of the cortex problem-those where $u(\phi)$ is even and those where it is odd. The general approach is the same in both cases-but the resulting planforms are different.

The generators of the planar lattices and their dual lattices that we use are given in table 1.

Table 1. Generators for the planar lattices and their dual lattices.

\begin{tabular}{lllll}
\hline Lattice & $\ell_{1}$ & $\ell_{2}$ & $k_{1}$ & $k_{2}$ \\
\hline Square & $(1,0)$ & $(0,1)$ & $(1,0)$ & $(0,1)$ \\
Hexagonal & $\left(1, \frac{1}{\sqrt{3}}\right)$ & $\left(0, \frac{2}{\sqrt{3}}\right)$ & $(1,0)$ & $\frac{1}{2}(-1, \sqrt{3})$ \\
Rhombic & $(1,-\cot \eta)$ & $(0, \operatorname{cosec} \eta)$ & $(1,0)$ & $(\cos \eta, \sin \eta)$ \\
\hline
\end{tabular}

Using lemma 3.1 and genericity we can write $\operatorname{ker} \mathrm{L}=Z(u)$. Moreover, we can write $Z(u)$ as a direct sum of rotated images of $Z_{u}$. Our explicit choices for this decomposition are listed in table 2 .

Table 2. Eigenfunctions corresponding to shortest dual wavevectors.

\begin{tabular}{ll}
\hline Lattice & ker $\mathrm{L}$ \\
\hline Square & $Z_{u} \oplus R_{\pi / 2}\left(Z_{u}\right)$ \\
Hexagonal & $Z_{u} \oplus R_{2 \pi / 3}\left(Z_{u}\right) \oplus R_{4 \pi / 3}\left(Z_{u}\right)$ \\
Rhombic & $Z_{u} \oplus R_{\eta}\left(Z_{u}\right)$ \\
\hline
\end{tabular}

\section{Coordinates on the irreducible subspace $Z(u)$}

We fix the function $u(\phi)$, which is real-valued, $\pi$-periodic and either even or odd. For the square and hexagonal lattices, we write the functions in $Z_{u}$ as $z_{1} u(\phi) \mathrm{e}^{2 \pi \mathrm{i} k_{1} \cdot x}+$ c.c., where $z_{1} \in \mathbb{C}$. The action of a rotation $R_{\theta}$ on $f(x, \phi)=u(\phi) \mathrm{e}^{2 \pi \mathrm{i} k \cdot x}$ is

$$
\left(R_{\theta} f\right)(\boldsymbol{x}, \phi)=f\left(R_{-\theta} \boldsymbol{x}, \phi-\theta\right)=u(\phi-\theta) \mathrm{e}^{2 \pi \mathrm{i} k \cdot R_{-\theta} x}=u(\phi-\theta) \mathrm{e}^{2 \pi \mathrm{i} R_{\theta} k \cdot \boldsymbol{x}} .
$$

On all lattices the action of rotation by $R_{\eta}$ (where $\eta=\frac{\pi}{2}$ on the square lattice and $\eta=\frac{2 \pi}{3}$ on the hexagonal lattice) shows that functions in $R_{\eta}\left(Z_{u}\right)$ have the form

$$
z_{2} R_{\eta}\left(u(\phi) \mathrm{e}^{2 \pi \mathrm{i} k_{1} \cdot x}+\text { c.c. }\right)=z_{2} u(\phi-\eta) \mathrm{e}^{2 \pi \mathrm{i} k_{2} \cdot x}+\text { c.c. }
$$

For the rhombic and square lattices the vectors $\left(z_{1}, z_{2}\right) \in \mathbb{C}^{2}$ span a real four-dimensional space. On the hexagonal lattice functions in $R_{4 \pi / 3}\left(Z_{u}\right)$ have the form

$$
z_{3} R_{4 \pi / 3}\left(u(\phi) \mathrm{e}^{2 \pi \mathrm{i} k_{1} \cdot x}\right)+\text { c.c. }=z_{3} u\left(\phi+\frac{2 \pi}{3}\right) \mathrm{e}^{2 \pi \mathrm{i} k_{3} \cdot x}+\text { c.c. }
$$


Here the vectors $\left(z_{1}, z_{2}, z_{3}\right) \in \mathbb{C}^{3}$ span a real six-dimensional space.

The form of the eigenfunctions in an irreducible representation corresponding to the shortest dual wavevectors in each lattice is given in table 3 .

Table 3. Eigenfunctions corresponding to $\Gamma_{\mathcal{L}}$ irreducible representation; $u(\phi)$ is $\pi$-periodic, realvalued and either odd or even.

\begin{tabular}{ll}
\hline Lattice & ker $\mathrm{L}$ \\
\hline Square & $z_{1} u(\phi) \mathrm{e}^{2 \pi \mathrm{i} k_{1} \cdot x}+z_{2} u\left(\phi-\frac{1}{2} \pi\right) \mathrm{e}^{2 \pi \mathrm{i} k_{2} \cdot x}+$ c.c. \\
Hexagonal & $z_{1} u(\phi) \mathrm{e}^{2 \pi \mathrm{i} k_{1} \cdot x}+z_{2} u\left(\phi-\frac{2}{3} \pi\right) \mathrm{e}^{2 \pi \mathrm{i} k_{2} \cdot x}+z_{3} u\left(\phi+\frac{2}{3} \pi\right) \mathrm{e}^{2 \pi \mathrm{i} k_{3} \cdot x}+$ c.c. \\
Rhombic & $z_{1} u(\phi) \mathrm{e}^{2 \pi \mathrm{i} k_{1} \cdot x}+z_{2} u(\phi-\eta) \mathrm{e}^{2 \pi \mathrm{i} k_{2} \cdot x}+$ c.c. \\
\hline
\end{tabular}

\section{Group actions on $Z(u)$}

There are two types of irreducible representation for each lattice corresponding to the cases $u(\phi)$ odd (pseudoscalars) and $u(\phi)$ even (scalars). We derive the explicit action of $\Gamma_{\mathcal{L}}$ on these subspaces.

The action of the torus $\boldsymbol{T}^{2}$ is derived as follows. Write $\theta \in \boldsymbol{T}^{2}$ as

$$
\theta=\theta_{1} \ell_{1}+\theta_{2} \ell_{2} \cong\left[\theta_{1}, \theta_{2}\right]
$$

where $0 \leqslant \theta_{j}<1$. The $\boldsymbol{T}^{2}$ action can be computed using the identity $\boldsymbol{k}_{i} \cdot \boldsymbol{\ell}_{j}=\delta_{i j}$. In particular,

$$
\theta\left(u(\phi) \mathrm{e}^{2 \pi \mathrm{i} k \cdot x}\right)=u(\phi) \mathrm{e}^{2 \pi \mathrm{i} k \cdot(x-\theta)}=\mathrm{e}^{-2 \pi \mathrm{i} k \cdot \theta} u(\phi) \mathrm{e}^{2 \pi \mathrm{i} k \cdot \boldsymbol{x}} .
$$

In coordinates on the square and rhombic lattices the torus action is

$$
\theta\left(z_{1}, z_{2}\right)=\left(\mathrm{e}^{-2 \pi \mathrm{i} \theta_{1}} z_{1}, \mathrm{e}^{-2 \pi \mathrm{i} \theta_{2}} z_{2}\right) .
$$

On the hexagonal lattice note that $\boldsymbol{k}_{3}=-\left(\boldsymbol{k}_{1}+\boldsymbol{k}_{2}\right)$. Thus the torus action is

$$
\theta\left(z_{1}, z_{2}, z_{3}\right)=\left(\mathrm{e}^{-2 \pi \mathrm{i} \theta_{1}} z_{1}, \mathrm{e}^{-2 \pi \mathrm{i} \theta_{2}} z_{2}, \mathrm{e}^{2 \pi \mathrm{i}\left(\theta_{1}+\theta_{2}\right)} z_{3}\right) .
$$

The holohedries $H_{\mathcal{L}}$ are $D_{4}, D_{6}$ and $D_{2}$ for the square, hexagonal and rhombic lattices, respectively. In each case the generators for these groups are a reflection and a rotation. For the square and hexagonal lattices, the reflection is $\kappa$. For the rhombic lattice, the reflection is $\kappa_{\eta}$, which interchanges $\boldsymbol{k}_{1}$ and $\boldsymbol{k}_{2}$. The counterclockwise rotation $\xi$, through angles $\frac{\pi}{2}, \frac{\pi}{3}$ and $\pi$, is the rotation generator for the three lattices. The actions of $H_{\mathcal{L}}$ are given in table 4 . Note that the two types of irreducible representation on the rhombic lattice are isomorphic. Thus, the solutions and their symmetries are identical. The patterns associated with these solutions, however, need not be the same in the two cases and they are different.

On the square lattice, the action of $\xi$ is

$$
\begin{aligned}
\xi\left(z_{1}, z_{2}\right) & \cong R_{\pi / 2}\left(z_{1} u(\phi) \mathrm{e}^{2 \pi \mathrm{i} k_{1} \cdot x}+z_{2} u\left(\phi-\frac{\pi}{2}\right) \mathrm{e}^{2 \pi \mathrm{i} k_{2} \cdot x}\right)+\text { c.c. } \\
& =z_{1} u\left(\phi-\frac{\pi}{2}\right) \mathrm{e}^{2 \pi \mathrm{i} k_{2} \cdot x}+z_{2} u(\phi) \mathrm{e}^{-2 \pi \mathrm{i} k_{1} \cdot x}+\text { c.c. } \\
& =\bar{z}_{2} u(\phi) \mathrm{e}^{2 \pi \mathrm{i} k_{1} \cdot x}+z_{1} u\left(\phi-\frac{\pi}{2}\right) \mathrm{e}^{2 \pi \mathrm{i} k_{2} \cdot x}+\text { c.c. } \\
& \cong\left(\bar{z}_{2}, z_{1}\right)
\end{aligned}
$$


Table 4. Left, $D_{2}+T^{2}$ action on rhombic lattice; centre, $D_{4}+T^{2}$ action on square lattice; right, $D_{6}+T^{2}$ action on hexagonal lattice. For $u(\phi)$ even, $\epsilon=+1$; for $u(\phi)$ odd, $\epsilon=-1$.

\begin{tabular}{llllll}
\hline $\boldsymbol{D}_{2}$ & Action & $\boldsymbol{D}_{4}$ & Action & $\boldsymbol{D}_{6}$ & Action \\
\hline $\mathbf{1}$ & $\left(z_{1}, z_{2}\right)$ & $\mathbf{1}$ & $\left(z_{1}, z_{2}\right)$ & $\mathbf{1}$ & $\left(z_{1}, z_{2}, z_{3}\right)$ \\
$\xi$ & $\left(\bar{z}_{1}, \bar{z}_{2}\right)$ & $\xi$ & $\left(\bar{z}_{2}, z_{1}\right)$ & $\xi$ & $\left(\bar{z}_{2}, \bar{z}_{3}, \bar{z}_{1}\right)$ \\
$\kappa_{\eta}$ & $\epsilon\left(z_{2}, z_{1}\right)$ & $\xi^{2}$ & $\left(\bar{z}_{1}, \bar{z}_{2}\right)$ & $\xi^{2}$ & $\left(z_{3}, z_{1}, z_{2}\right)$ \\
$\kappa_{\eta} \xi$ & $\epsilon\left(\bar{z}_{2}, \bar{z}_{1}\right)$ & $\xi^{3}$ & $\left(z_{2}, \bar{z}_{1}\right)$ & $\xi^{3}$ & $\left(\bar{z}_{1}, \bar{z}_{2}, \bar{z}_{3}\right)$ \\
& & $\kappa$ & $\epsilon\left(z_{1}, \bar{z}_{2}\right)$ & $\xi^{4}$ & $\left(z_{2}, z_{3}, z_{1}\right)$ \\
& & $\kappa \xi$ & $\epsilon\left(\bar{z}_{2}, \bar{z}_{1}\right)$ & $\xi^{5}$ & $\left(\bar{z}_{3}, \bar{z}_{1}, \bar{z}_{2}\right)$ \\
& & $\kappa \xi^{2}$ & $\epsilon\left(\bar{z}_{1}, z_{2}\right)$ & $\kappa$ & $\epsilon\left(z_{1}, z_{3}, z_{2}\right)$ \\
& & $\kappa \xi^{3}$ & $\epsilon\left(z_{2}, z_{1}\right)$ & $\kappa \xi$ & $\epsilon\left(\bar{z}_{2}, \bar{z}_{1}, \bar{z}_{3}\right)$ \\
& & & $\kappa \xi^{2}$ & $\epsilon\left(z_{3}, z_{2}, z_{1}\right)$ \\
& & & $\kappa \xi^{3}$ & $\epsilon\left(\bar{z}_{1}, \bar{z}_{3}, \bar{z}_{2}\right)$ \\
& & & $\kappa \xi^{4}$ & $\epsilon\left(z_{2}, z_{1}, z_{3}\right)$ \\
& & & $\kappa \xi^{5}$ & $\epsilon\left(\bar{z}_{3}, \bar{z}_{2}, \bar{z}_{1}\right)$ \\
$\left.\theta_{1}, \theta_{2}\right]$ & & & $\left(\mathrm{e}^{-2 \pi \mathrm{i} \theta_{1}} z_{1}, \mathrm{e}^{-2 \pi \mathrm{i} \theta_{2}} z_{2}\right)$ & $\left(\mathrm{e}^{-2 \pi \mathrm{i} \theta_{1}} z_{1}, \mathrm{e}^{-2 \pi \mathrm{i} \theta_{2}} z_{2}, \mathrm{e}^{2 \pi \mathrm{i}\left(\theta_{1}+\theta_{2}\right)} z_{3}\right)$ \\
\hline
\end{tabular}

and the action of $\kappa$ is

$$
\begin{aligned}
\kappa\left(z_{1}, z_{2}\right) & \cong \kappa\left(z_{1} u(\phi) \mathrm{e}^{2 \pi \mathrm{i} k_{1} \cdot x}+z_{2} u\left(\phi-\frac{\pi}{2}\right) \mathrm{e}^{2 \pi \mathrm{i} k_{2} \cdot x}\right)+\text { c.c. } \\
& =z_{1} u(-\phi) \mathrm{e}^{2 \pi \mathrm{i} k_{1} \cdot x}+z_{2} u\left(\frac{\pi}{2}-\phi\right) \mathrm{e}^{-2 \pi \mathrm{i} k_{2} \cdot x}+\text { c.c. } \\
& = \pm\left(z_{1} u(\phi) \mathrm{e}^{2 \pi \mathrm{i} k_{1} \cdot x}+\bar{z}_{2} u\left(\phi-\frac{\pi}{2}\right) \mathrm{e}^{2 \pi \mathrm{i} k_{2} \cdot x}\right)+\text { c.c. } \\
& \cong \pm\left(z_{1}, \bar{z}_{2}\right) .
\end{aligned}
$$

On the hexagonal lattice the action of $\xi$ is

$$
\begin{aligned}
\xi\left(z_{1}, z_{2}, z_{3}\right) \cong & R_{\pi / 3}\left(z_{1} u(\phi) \mathrm{e}^{2 \pi \mathrm{i} k_{1} \cdot x}+z_{2} u\left(\phi-\frac{2 \pi}{3}\right) \mathrm{e}^{2 \pi \mathrm{i} k_{2} \cdot x}\right. \\
& \left.+z_{3} u\left(\phi+\frac{2 \pi}{3}\right) \mathrm{e}^{2 \pi \mathrm{i} k_{3} \cdot x}\right)+ \text { c.c. } \\
= & z_{1} u\left(\phi-\frac{\pi}{3}\right) \mathrm{e}^{-2 \pi \mathrm{i} k_{3} \cdot x}+z_{2} u(\phi-\pi) \mathrm{e}^{-2 \pi \mathrm{i} k_{1} \cdot x}+z_{3} u\left(\phi+\frac{\pi}{3}\right) \mathrm{e}^{-2 \pi \mathrm{i} k_{2} \cdot x}+\text { c.c. } \\
= & \bar{z}_{2} u(\phi) \mathrm{e}^{2 \pi \mathrm{i} k_{1} \cdot x}+\bar{z}_{3} u\left(\phi-\frac{2 \pi}{3}\right) \mathrm{e}^{2 \pi \mathrm{i} k_{2} \cdot x}+\bar{z}_{1} u\left(\phi+\frac{2 \pi}{3}\right) \mathrm{e}^{2 \pi \mathrm{i} k_{3} \cdot x}+\text { c.c. } \\
\cong & \left(\bar{z}_{2}, \bar{z}_{3}, \bar{z}_{1}\right)
\end{aligned}
$$

using the $\pi$-periodicity of $u$. The action of $\kappa$ is

$$
\begin{aligned}
\kappa\left(z_{1}, z_{2}, z_{3}\right) & \cong \kappa\left(z_{1} u(\phi) \mathrm{e}^{2 \pi \mathrm{i} k_{1} \cdot x}+z_{2} u\left(\phi-\frac{2 \pi}{3}\right) \mathrm{e}^{2 \pi \mathrm{i} k_{2} \cdot x}+z_{3} u\left(\phi+\frac{2 \pi}{3}\right) \mathrm{e}^{2 \pi \mathrm{i} k_{3} \cdot x}\right)+\text { c.c. } \\
& =z_{1} u(-\phi) \mathrm{e}^{2 \pi \mathrm{i} k_{1} \cdot x}+z_{2} u\left(\frac{2 \pi}{3}-\phi\right) \mathrm{e}^{2 \pi \mathrm{i} k_{3} \cdot x}+z_{3} u\left(\frac{2 \pi}{3}-\phi\right) \mathrm{e}^{2 \pi \mathrm{i} k_{2} \cdot x}+\text { c.c. } \\
& \cong \pm\left(z_{1}, z_{3}, z_{2}\right) .
\end{aligned}
$$


On the rhombic lattice the action of $\xi$ is

$$
\begin{aligned}
\xi\left(z_{1}, z_{2}\right) & \cong R_{\pi}\left(z_{1} u(\phi) \mathrm{e}^{2 \pi \mathrm{i} k_{1} \cdot x}+z_{2} u(\phi-\eta) \mathrm{e}^{2 \pi \mathrm{i} k_{2} \cdot x}\right)+\text { c.c. } \\
& \left.=z_{1} u(\phi-\pi) \mathrm{e}^{-2 \pi \mathrm{i} k_{1} \cdot x}+z_{2} u(\phi-\eta-\pi) \mathrm{e}^{-2 \pi \mathrm{i} k_{2} \cdot x}\right)+ \text { c.c. } \\
& \cong\left(\bar{z}_{1}, \bar{z}_{2}\right) .
\end{aligned}
$$

The action of $\kappa_{\eta}$ on $\mathbb{R}^{2} \times S^{1}$ is $(x, \phi) \mapsto\left(\kappa_{\eta} x, \eta-\phi\right)$ and the lattice action is

$$
\begin{aligned}
\kappa_{\eta}\left(z_{1}, z_{2}\right) & \cong \kappa_{\eta}\left(z_{1} u(\phi) \mathrm{e}^{2 \pi \mathrm{i} k_{1} \cdot x}+z_{2} u(\phi-\eta) \mathrm{e}^{2 \pi \mathrm{i} k_{2} \cdot x}\right)+\text { c.c. } \\
& =z_{1} u(\eta-\phi) \mathrm{e}^{2 \pi \mathrm{i} k_{2} \cdot x}+z_{2} u(-\phi) \mathrm{e}^{2 \pi \mathrm{i} k_{1} \cdot x}+\text { c.c. } \\
& \cong \pm\left(z_{2}, z_{1}\right) .
\end{aligned}
$$

Remark 3.2. Note that the scalar and pseudoscalar actions are identical on the square and rhombic lattices. This assertion is verified by noting that the torus element $\theta=\left[\frac{1}{2}, \frac{1}{2}\right]$ acts by $\left(z_{1}, z_{2}\right) \mapsto\left(-z_{1},-z_{2}\right)$. It follows that $\theta \kappa$ acts in the pseudoscalar representation exactly as $\kappa$ acts in the scalar representation. Thus the two representations are generated by the same set of matrices on $\mathbb{C}^{2}$. The planforms for the two actions will be different since the functions $u(\phi)$ are different in the two cases. Moreover, as we shall see, the symmetry groups of the planforms are different (though isomorphic as groups). The corresponding statement is false for the hexagonal lattice where the scalar and pseudoscalar actions are not isomorphic.

\section{The axial subgroups}

For each of the six irreducible representations, we compute the axial subgroups, i.e. those isotropy subgroups that have one-dimensional fixed-point subspaces. The computations for the even (scalar) case are well known (see [5] for the hexagonal lattice). The odd (pseudoscalar) case was introduced by Bosch Vivancos et al [1]. In each case it is an easy exercise to use table 4 to verify that the listed subgroups are indeed axial subgroups.

Showing that the listed subgroups are, up to conjugacy, all of the axial subgroups is straightforward for the square and rhombic lattices. Note that we can use the $T^{2}$-action to assume, after conjugacy, that $z_{1}$ and $z_{2}$ are real and non-negative. The results are listed in tables 5, 7, 8 and 10. The computations on the hexagonal lattice are more complicated; the results are listed in tables 6 and 9 . We present the details of the classification in the odd case on the hexagonal lattice.

Table 5. Axial subgroups for square lattice: $u(-\phi)=u(\phi)$ even, $\boldsymbol{x}=(x, y)$.

\begin{tabular}{ll}
\hline Axial & Planform eigenfunction \\
\hline Esquares & $u(\phi) \cos (2 \pi x)+u\left(\phi-\frac{1}{2} \pi\right) \cos (2 \pi y)$ \\
Erolls & $u(\phi) \cos (2 \pi x)$ \\
\hline
\end{tabular}

Table 6. Axial subgroups for hexagonal lattice: $u(-\phi)=u(\phi)$ even.

\begin{tabular}{ll}
\hline Axial & Planform eigenfunction \\
\hline EhexagonsP & $u(\phi) \cos (2 \pi x)+u\left(\phi-\frac{2}{3} \pi\right) \cos \left(2 \pi \boldsymbol{k}_{2} \cdot \boldsymbol{x}\right)+u\left(\phi+\frac{2}{3} \pi\right) \cos \left(2 \pi \boldsymbol{k}_{3} \cdot \boldsymbol{x}\right)$ \\
EhexagonsM & $-u(\phi) \cos (2 \pi x)-u\left(\phi-\frac{2}{3} \pi\right) \cos \left(2 \pi \boldsymbol{k}_{2} \cdot \boldsymbol{x}\right)-u\left(\phi+\frac{2}{3} \pi\right) \cos \left(2 \pi \boldsymbol{k}_{3} \cdot \boldsymbol{x}\right)$ \\
Erolls & $u(\phi) \cos (2 \pi x)$ \\
\hline
\end{tabular}


Table 7. Axial subgroups for rhombic lattice: $u(-\phi)=-u(\phi)$ even.

\begin{tabular}{ll}
\hline Axial & Planform eigenfunction \\
\hline Erhombs & $u(\phi) \cos (2 \pi x)+u(\eta-\phi) \cos \left(2 \pi \boldsymbol{k}_{2} \cdot \boldsymbol{x}\right)$ \\
Erolls & $u(\phi) \cos (2 \pi x)$ \\
\hline
\end{tabular}

Table 8. Axial subgroups for square lattice: $u(-\phi)=-u(\phi)$ odd.

\begin{tabular}{ll}
\hline Axial & Planform eigenfunction \\
\hline Osquares & $u(\phi) \cos (2 \pi x)+u\left(\phi-\frac{1}{2} \pi\right) \cos (2 \pi y)$ \\
Orolls & $u(\phi) \cos (2 \pi x)$ \\
\hline
\end{tabular}

Table 9. Axial subgroups for hexagonal lattice: $u(-\phi)=-u(\phi)$ odd .

\begin{tabular}{ll}
\hline Axial & Planform eigenfunction \\
\hline Ohexagons & $u(\phi) \cos (2 \pi x)+u\left(\phi-\frac{2}{3} \pi\right) \cos \left(2 \pi \boldsymbol{k}_{2} \cdot \boldsymbol{x}\right)+u\left(\phi+\frac{2}{3} \pi\right) \cos \left(2 \pi \boldsymbol{k}_{3} \cdot \boldsymbol{x}\right)$ \\
Otriangles & $u(\phi) \sin (2 \pi x)+u\left(\phi-\frac{2}{3} \pi\right) \sin \left(2 \pi \boldsymbol{k}_{2} \cdot \boldsymbol{x}\right)+u\left(\phi+\frac{2}{3} \pi\right) \sin \left(2 \pi \boldsymbol{k}_{3} \cdot \boldsymbol{x}\right)$ \\
Orectangles & $u\left(\phi-\frac{2}{3} \pi\right) \cos \left(2 \pi \boldsymbol{k}_{2} \cdot \boldsymbol{x}\right)-u\left(\phi+\frac{2}{3} \pi\right) \cos \left(2 \pi \boldsymbol{k}_{3} \cdot \boldsymbol{x}\right)$ \\
Orolls & $u(\phi) \cos (2 \pi x)$ \\
\hline
\end{tabular}

Table 10. Axial subgroups for rhombic lattice: $u(-\phi)=-u(\phi)$ odd.

\begin{tabular}{ll}
\hline Axial & Planform eigenfunction \\
\hline Orhombs & $u(\phi) \cos (2 \pi x)+u(\eta-\phi) \cos \left(2 \pi \boldsymbol{k}_{2} \cdot \boldsymbol{x}\right)$ \\
Orolls & $u(\phi) \cos (2 \pi x)$ \\
\hline
\end{tabular}

To summarize we list all of the planforms by name and isotropy subgroup in table 11 . We also include the element from the fixed-point subspace of the isotropy subgroup that generates the eigenfunctions listed in tables 5-10.

\subsection{The hexagonal lattice: odd case}

The results for the odd case on the hexagonal lattice are reminiscent of the planform results on the hexagonal lattice for the Boussinesq equations with the midplane reflection (see $[8,9])$ in that there are four axial subgroups in each case, but there are slight differences in the symmetries of solutions in these two cases.

Lemma 4.1. On the hexagonal lattice in the odd case each of the four subgroups listed in table 9 are axial subgroups. Up to conjugacy, these are the only axial subgroups.

Proof. We verify that up to conjugacy these are the only axial subgroups. Our method is to conjugate elements in $z=\left(z_{1}, z_{2}, z_{3}\right) \in \mathbb{C}^{3}$ by elements in $\Gamma_{\mathcal{L}}$ (conjugate points have conjugate isotropy subgroups) in a way that allows us to enumerate all isotropy subgroups (up to conjugacy) and thus list all axial subgroups.

After an application of $\xi^{2}$ and $\kappa$ if necessary, we can assume that

$$
\left|z_{1}\right| \leqslant\left|z_{2}\right| \leqslant\left|z_{3}\right| .
$$

We consider four cases:
$\left|z_{1}\right|<\left|z_{2}\right|<\left|z_{3}\right|$
$\left|z_{1}\right|<\left|z_{2}\right|=\left|z_{3}\right|$
$\left|z_{1}\right|=\left|z_{2}\right|<\left|z_{3}\right|$
$\left|z_{1}\right|=\left|z_{2}\right|=\left|z_{3}\right|$. 
Table 11. A summary of the axial subgroups. $\boldsymbol{O}(2)$ is generated by $\left[0, \theta_{2}\right] \in \boldsymbol{T}^{2}$ and rotation by $\pi$ ( $\xi$ on a rhombic lattice, $\xi^{2}$ on a square lattice and $\xi^{3}$ on a hexagonal lattice). On the even hexagonal lattice the points $(1,1,1)$ and $(-1,-1,-1)$ have the same isotropy subgroup $\left(\boldsymbol{D}_{6}\right)$, but are not conjugate by a group element. Therefore, the associated eigenfunctions generate different planforms.

\begin{tabular}{llll}
\hline Lattice & Axial & Isotropy subgroup & Fixed vector \\
\hline Square & Esquares & $\boldsymbol{D}_{4}(\kappa, \xi)$ & $(1,1)$ \\
& Erolls & $\boldsymbol{O}(2) \oplus \mathbb{Z}_{2}(\kappa)$ & $(1,0)$ \\
Hexagonal & EhexagonsP & $\boldsymbol{D}_{6}$ & $(1,1,1)$ \\
& EhexagonsM & $\boldsymbol{D}_{6}$ & $(-1,-1,-1)$ \\
& Erolls & $\boldsymbol{O}(2) \oplus \mathbb{Z}_{2}(\kappa)$ & $(1,0,0)$ \\
Rhombic & Erhombs & $\boldsymbol{D}_{2}\left(\kappa_{\eta}, \xi\right)$ & $(1,1)$ \\
& Erolls & $\boldsymbol{O}(2)$ & $(1,0)$ \\
Square & Osquares & $\boldsymbol{D}_{4}\left(\kappa\left[\frac{1}{2}, \frac{1}{2}\right], \xi\right)$ & $(1,1)$ \\
& Orolls & $\boldsymbol{O}(2) \oplus \mathbb{Z}_{2}\left(\xi^{2} \kappa\left[\frac{1}{2}, 0\right]\right)$ & $(1,0)$ \\
Hexagonal & Ohexagons & $\mathbb{Z}_{6}$ & $(1,1,1)$ \\
& Otriangles & $\boldsymbol{D}_{3}\left(\kappa \xi, \xi^{2}\right)$ & $(\mathrm{i}, \mathrm{i}, \mathrm{i})$ \\
& Orectangles & $\boldsymbol{D}_{2}\left(\kappa, \xi^{3}\right)$ & $(0,1,-1)$ \\
& Orolls & $\boldsymbol{O}(2) \oplus \mathbb{Z}_{4}\left(\xi^{3} \kappa\left[\frac{1}{2}, 0\right]\right)$ & $(1,0,0)$ \\
Rhombic & Orhombs & $\boldsymbol{D}_{2}\left(\kappa_{\eta}\left[\frac{1}{2}, \frac{1}{2}\right], \xi\right)$ & $(1,1)$ \\
& Orolls & $\boldsymbol{O}(2)$ & $(1,0)$ \\
\hline
\end{tabular}

$\left|z_{1}\right|<\left|z_{2}\right|<\left|z_{3}\right|$. Since elements in $\boldsymbol{D}_{6}$ permute the $z_{j}$ and elements of $\boldsymbol{T}^{2}$ preserve coordinates, it follows that symmetries of these points can only involve elements in $\boldsymbol{D}_{6}$ that preserve coordinates; that is, $\mathbf{1}$ and $\xi^{3}$. After conjugacy by a translation, we can assume that $z_{2}=x_{2}>0$ and $z_{3}=x_{3}>0$.

It follows from table 4 that if $\theta \in T^{2}$ fixes $z$, then $\theta=0$. Therefore, there are two possible isotropy subgroups: $\mathbb{Z}_{2}\left(\xi^{3}\right)$ when $z_{1} \in \mathbb{R}$ and $\mathbf{1}$ when $z_{1} \notin \mathbb{R}$.

$\left|z_{1}\right|<\left|z_{2}\right|=\left|z_{3}\right|$. Conjugate $z$ by an element of $\boldsymbol{T}^{2}$ so that $z_{2}=x>0$ and $z_{3}=-x$. The previous calculation shows that no non-identity torus element fixes $z$. The collection of points $\left(z_{1}, x,-x\right)$ is preserved only by $\xi^{3}, \kappa$ and $\kappa \xi^{3}$. In particular,

$$
\begin{aligned}
& \xi^{3}\left(z_{1}, x,-x\right)=\left(\bar{z}_{1}, x,-x\right) \\
& \kappa\left(z_{1}, x,-x\right)=\left(-z_{1}, x,-x\right) .
\end{aligned}
$$

Let $z_{1}=x_{1}+\mathrm{i} y_{1}$. Using $\xi^{3}$ and $\kappa$ we can assume $x_{1} \geqslant 0$ and $y_{1} \geqslant 0$. If $x_{1}, y_{1}>0$, the isotropy subgroup is $\mathbf{1}$. If $x_{1} \neq 0, y_{1}=0$, then the isotropy subgroup is $\mathbb{Z}_{2}\left(\xi^{3}\right)$. If $x_{1}=0, y_{1} \neq 0$, then the isotropy subgroup is $\mathbb{Z}_{2}\left(\xi^{3} \kappa\right)$. If $z_{1}=0$, then the isotropy subgroup is $\boldsymbol{D}_{2}\left(\xi^{3}, \kappa\right)$.

$\left|z_{1}\right|=\left|z_{2}\right|<\left|z_{3}\right|$. When $z_{1}>0$ this case is the same as the previous one. We consider the possibility $z_{1}=z_{2}=0$. In this case, $z$ is conjugate to $(0,0, x)$, which in turn is conjugate to $(x, 0,0)$. Note that

$$
\kappa\left[\frac{1}{2}, 0\right]\left(z_{1}, z_{2}, z_{3}\right)=\left(z_{1}, z_{3},-z_{2}\right)
$$

is an order four element. The isotropy subgroup of $(x, 0,0)$ is generated by

$$
\left[0, \theta_{2}\right] \quad \xi^{3} \quad \kappa\left[\frac{1}{2}, 0\right] .
$$


Table 12. Isotropy subgroups for pseudoscalar action of $D_{6}+T^{2}$ up to conjugacy.

\begin{tabular}{llll}
\hline Name & Isotropy subgroup $\Sigma$ & $\operatorname{Fix}(\Sigma)$ & $\operatorname{dim} \operatorname{Fix}(\Sigma)$ \\
\hline \multirow{3}{*}{ Orolls } & $\boldsymbol{D}_{6}+\boldsymbol{T}^{2}$ & 0 & 0 \\
Otriangles & $\boldsymbol{O}(2)\left(\left[0, \theta_{2}\right], \xi^{3}\right) \times \mathbb{Z}_{4}(\rho)$ & $(x, 0,0)$ & 1 \\
Ohexagons & $\boldsymbol{D}_{3}\left(\xi^{2}, \kappa \xi\right)$ & $(y \mathrm{i}, y \mathrm{i}, y \mathrm{i})$ & 1 \\
Orectangles & $\mathbb{Z}_{6}(\xi)$ & $(x, x, x)$ & 1 \\
& $\boldsymbol{D}_{2}\left(\xi^{3}, \kappa\right)$ & $\left(0, x_{2},-x_{2}\right)$ & 1 \\
& $\mathbb{Z}_{2}\left(\xi^{3}\right)$ & $\left(x_{1}, x_{2}, x_{3}\right)$ & 3 \\
& $\mathbb{Z}_{2}\left(\xi^{3} \kappa\right)$ & $\left(y_{1} \mathrm{i}, z_{2},-\bar{z}_{2}\right)$ & 3 \\
& $\mathbf{1}$ & $\left(z_{1}, z_{2}, z_{3}\right)$ & 6 \\
\hline
\end{tabular}

Together $\left[0, \theta_{2}\right]$ and $\xi^{3}$ generate the group $\boldsymbol{O}(2)$ and

$$
\rho=\xi^{3} \kappa\left[\frac{1}{2}, 0\right]
$$

is an order four element that commutes with $\boldsymbol{O}(2)$. Therefore, the isotropy subgroup is $\boldsymbol{O}(2) \times \mathbb{Z}_{4}(\rho)$.

$\left|z_{1}\right|=\left|z_{2}\right|=\left|z_{3}\right| . \quad$ If $z=0$, then the isotropy subgroup is $\Gamma_{\mathcal{L}}$. If not, as above, we can use $T^{2}$ to conjugate to $z_{2}=1$ and $z_{3}=-1$. Also, as above, we can conjugate $z_{1}=x_{1}+\mathrm{i} y_{1}$ so that $x_{1}, y_{1} \geqslant 0$. Since $\left|z_{1}\right|=1$, there are three cases $z_{1}=1, z_{1}=\mathrm{i}$ and $z_{1}$ neither real nor imaginary. In the first case $z=(1,1,-1)$ is conjugate (by $\left.\left[0,-\frac{1}{4}\right]\right)$ to $(1,1,1)$, whose isotropy subgroup is $\mathbb{Z}_{6}(\xi)$. In the second case (i, $\left.1,-1\right)$ is conjugate (by $\left.\kappa\left[\frac{1}{2}, \frac{1}{2}\right]\right)$ to $(\mathrm{i}, \mathrm{i}, \mathrm{i}$ ), whose isotropy subgroup is $D_{3}\left(\xi^{2}, \kappa \xi\right)$. Finally, points $z=\left(z_{1}, 1,-1\right)$ with $\left|z_{1}\right|=1$ and $z_{1}$ neither real nor purely imaginary have a trivial isotropy subgroup.

\subsection{Pictures of the axial planforms}

We picture the planforms by associating a line field to $f(x, \phi)$ as follows. For each $\boldsymbol{x}$, choose the direction $\phi$ that maximizes the function $f(\boldsymbol{x}, \cdot)$ on the circle $\boldsymbol{S}^{1}$. Note that this process leads to a line field with discontinuities at points $\boldsymbol{x}$ where $f$ has more than one absolute maximum. In the figures, we graph a small line element in the direction $\phi$ for a grid of points $x$. Where double maxima occur, we have attempted to draw all directions having this maximum value. The raw pattern of activity in V1 described by a line field needs additional processing before it can be interpreted as a hallucinatory visual image. Such processing involves a coarse-graining of the cortical activity pattern followed by a mapping to visual field coordinates as detailed in [2].

In the eigenfunctions choose the arbitrary $\pi$-periodic function $u(\phi)$ as follows. Expand $u$ as a Fourier series. In the odd case, it seems reasonable to choose just the first non-zero term $u(\phi)=\sin (2 \phi)$. This choice, however, is non-generic since equal maxima occur only when the eigenfunction is identically zero. To verify this assertion observe, for example, that the eigenfunction for odd hexagons

$\mathrm{e}^{2 \pi \mathrm{i} k_{1} \cdot x} \sin (2 \phi)+\mathrm{e}^{2 \pi \mathrm{i} k_{2} \cdot x} \sin \left(2\left(\phi-\frac{2 \pi}{3}\right)\right)+\mathrm{e}^{2 \pi \mathrm{i} k_{3} \cdot x} \sin \left(2\left(\phi+\frac{2 \pi}{3}\right)\right)+$ c.c.

can be transformed to

$$
A(x) \cos \left(2\left(\phi-\phi_{0}(x)\right)\right) .
$$


In this form maxima can occur only when $A(x)=0$ or $\phi=\phi_{0}(x)$. For a given $x$, the latter value of $\phi$ is unique. So multiple maxima occur only when $A(x)=0$, that is, when the eigenfunction is identically zero.

To avoid this source of non-genericity, we perform our calculations using $u(\phi)=$ $\sin (2 \phi)-0.5 \sin (4 \phi)$. In the even case, the first non-zero term is the constant term, which has no effect when choosing maxima. So, in the even case, we choose the function $u(\phi)=\cos (2 \phi)-0.5 \cos (4 \phi)$. An explicit calculation of eigenfunctions for the WilsonCowan equations is carried out in [2] using a perturbation expansion in the coupling strength of lateral connections.

\section{The structure of discontinuities in planform line fields}

Let $f(\boldsymbol{x}, \phi)$ be an eigenfunction and $\mathcal{X}_{f}$ be the points $\boldsymbol{x}$ where $f(\boldsymbol{x}, \cdot)$ has multiple absolute maxima. The set $\mathcal{X}_{f}$ is the set of points where the line field planform has discontinuities. Note that $\mathcal{X}_{f}$ is a codimension-one algebraic variety; that is, $\mathcal{X}_{f}$ is a collection of (perhaps intersecting) curves that divides the plane into cells. On each cell, the line field varies smoothly and, in the examples we consider, is, more or less, constant. Moreover, suppose that $\sigma \in \Gamma_{\mathcal{L}}$. Then $\sigma$ acts both on $x$ and $\phi$. We assert that if $\sigma$ is a symmetry of an eigenfunction $f(x, \phi)$ then $\sigma \mathcal{X}_{f}=\mathcal{X}_{f}$, that is, the set $\mathcal{X}_{f}$ is invariant under the isotropy subgroup of the eigenfunction. For example, this remark allows us to see a difference in planforms between $\boldsymbol{D}_{6}$ hexagons on the even hexagonal lattice and $\mathbb{Z}_{6}$ hexagons on the odd hexagonal lattice.

The symmetries of individual solutions give some hint about discontinuities in the associated line fields. For example, suppose that $f(\boldsymbol{x}, \phi)$ is invariant under the reflection $\kappa$. Then, $f\left(x_{1},-x_{2},-\phi\right)=f\left(x_{1}, x_{2}, \phi\right)$. It follows that $f\left(x_{1}, 0,-\phi\right)=f\left(x_{1}, 0, \phi\right)$. Therefore, the maximum value of $f\left(x_{1}, 0, \cdot\right)$ can be unique only when that maximum occurs at $\phi=0$ or $\phi=\frac{\pi}{2}$. If $f$ is an even roll solution, then whole stripes are forced to have multiple maxima, as illustrated in figure 2 .

These remarks are valid for solutions to the full nonlinear equations-not just for the particular eigenfunctions that we have chosen for illustrative purposes.

\section{Planform selection: a review of previous work}

In section 4 we listed all axial subgroups on the rhombic, square and hexagonal lattices in both the scalar and pseudoscalar cases corresponding to critical wavevectors of shortest length in the dual lattice. We also illustrated our findings using line field pictures associated with eigenfunctions of the visual cortex model. In this section we address the question of stability of these planforms and, where relevant, secondary bifurcations. Stability is a difficult issue and we address only one part of this complex problem.

Fix a planar lattice $\mathcal{L}$. We answer the following question. Determine the stability of axial solutions to perturbations that are doubly periodic with respect to $\mathcal{L}$. As discussed in section 1 we can find solutions near bifurcation by reducing the original differential equations model to a system of ordinary differential equations on a centre manifold. Moreover, centre manifold reduction can be done in a way that preserves the symmetries of the system. Existence of equilibria for the centre manifold equations is found using the equivariant branching lemma. To determine stability, we need to discuss the structure of $\Gamma_{\mathcal{L}^{-}}$-equivariant vector fields and isotypic decompositions of the associated Jacobian matrices at equilibrium points.

Except for the pseudoscalar representation on the hexagonal lattice, the information has been computed in a number of different venues. For completeness, we begin by sketching the previously known material. In each case we assume that $f(z)$ is a $\Gamma_{\mathcal{L}}$-equivariant mapping. 

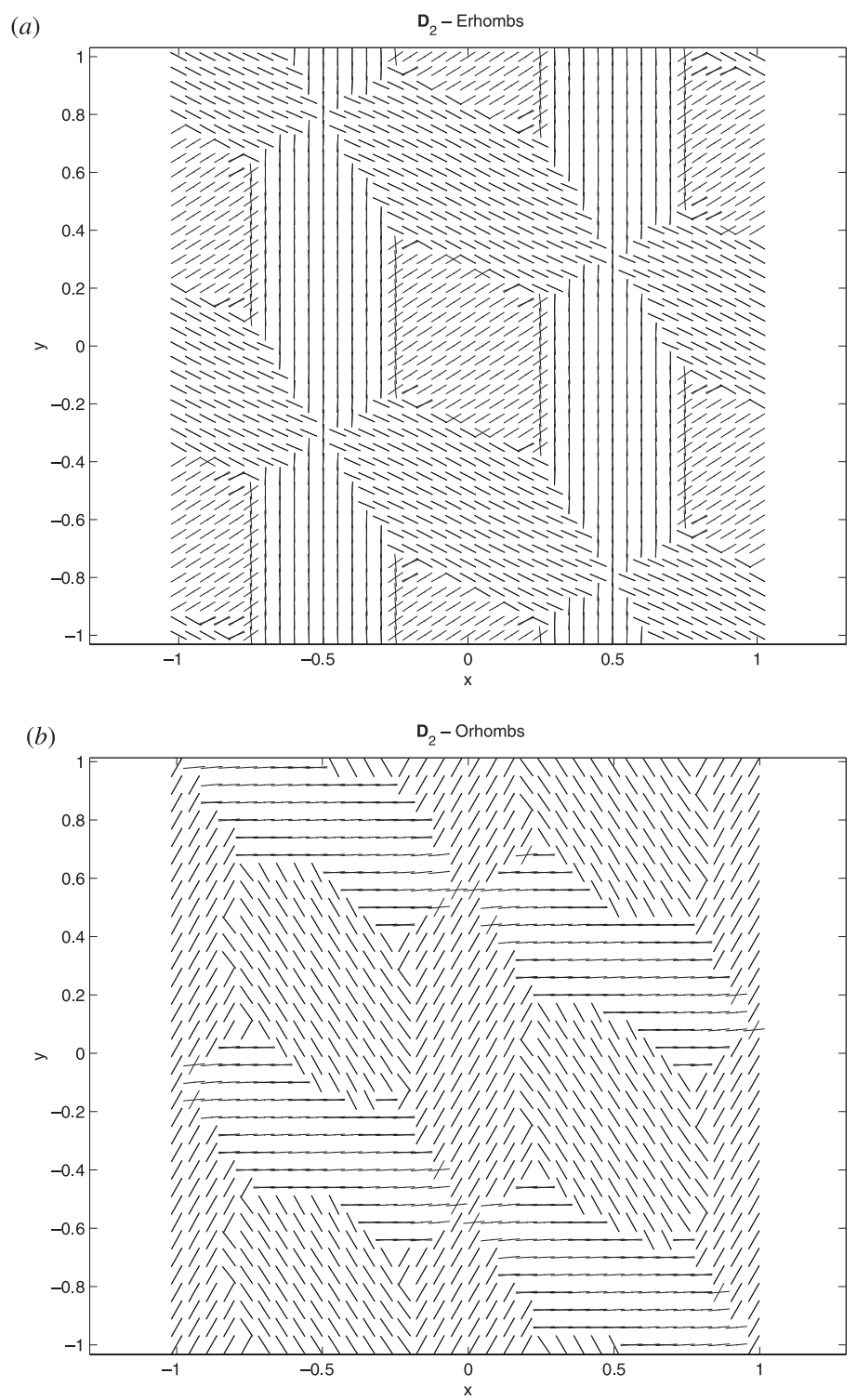

Figure 2. Rhombic lattice $\left(\eta=\frac{\pi}{2.8}\right)$ : (a) even rhombs, $(b)$ odd rhombs, $(c)$ even rolls, $(d)$ odd rolls.

These bifurcation analyses are used in [2] where $f$ has been computed to third order for both scalar and pseudoscalar representations by perturbation expansions in models based on the Wilson-Cowan equations [13].

\section{The rhombic lattice}

The scalar and pseudoscalar actions of $\Gamma_{\mathcal{L}}$ on $\mathbb{C}^{2}$ are identical in the sense that the two actions generate precisely the same set of linear mappings on $\mathbb{C}^{2}$ (see remark 3.2). Therefore, the two actions produce the same set of equivariant mappings and the same abstract bifurcation theory. 
(c)
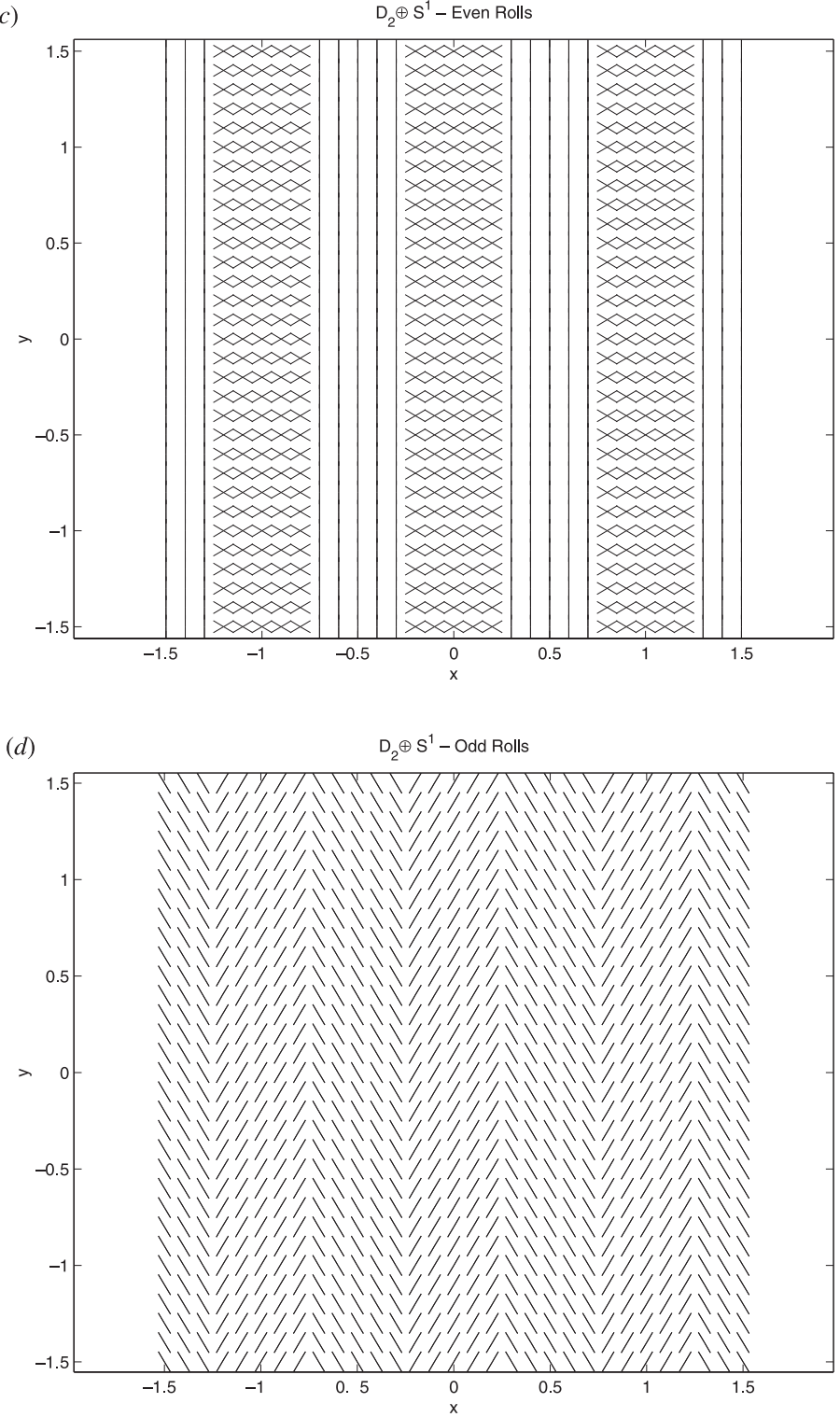

Figure 2. Continued.

Lemma 5.1. The general equivariant mapping on the rhombic lattice shortest wavevector representation is

$$
f\left(z_{1}, z_{2}\right)=\left(p\left(\left|z_{1}\right|^{2},\left|z_{2}\right|^{2}\right) z_{1}, p\left(\left|z_{2}\right|^{2},\left|z_{1}\right|^{2}\right) z_{2}\right)
$$

where $p$ is real-valued and odd.

Proof. Let $f: \mathbb{C}^{2} \rightarrow \mathbb{C}^{2}$ be $\Gamma_{\mathcal{L}}$-equivariant. Commutativity with respect to the torus action implies that

$$
f\left(z_{1}, z_{2}\right)=\left(P\left(\left|z_{1}\right|^{2},\left|z_{2}\right|^{2}\right) z_{1}, Q\left(\left|z_{1}\right|^{2},\left|z_{2}\right|^{2}\right) z_{2}\right)
$$



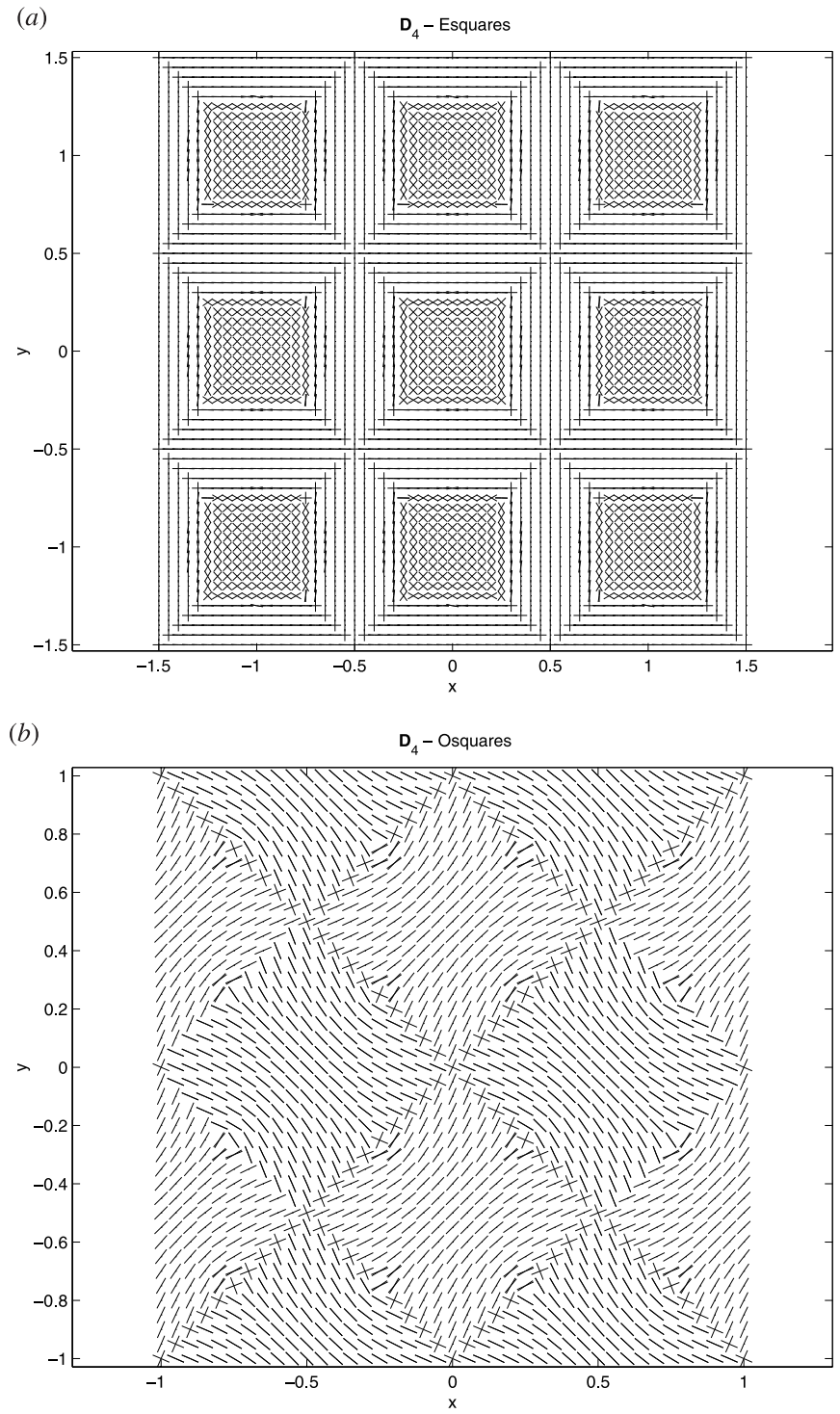

Figure 3. Square lattice: $(a)$ even squares and $(b)$ odd squares.

for some complex-valued functions $P$ and $Q$. Commutativity with respect to $\xi z=\bar{z}$ implies that $P$ and $Q$ are real-valued. Indeed, $f$ is determined by the mapping $g: \mathbb{R}^{2} \rightarrow \mathbb{R}^{2}$ of the form

$$
g\left(x_{1}, x_{2}\right)=\left(p\left(x_{1}^{2}, x_{2}^{2}\right) x_{1}, q\left(x_{1}^{2}, x_{2}^{2}\right) x_{2}\right) .
$$

Moreover, $g$ has $\boldsymbol{D}_{2}$ symmetry, since it commutes with $\left(x_{1}, x_{2}\right) \mapsto\left(x_{2}, x_{1}\right)$ and $x \mapsto-x$. Therefore,

$$
g\left(x_{1}, x_{2}\right)=\left(p\left(x_{1}^{2}, x_{2}^{2}\right) x_{1}, p\left(x_{2}^{2}, x_{1}^{2}\right) x_{2}\right)
$$

where $p$ is an odd function. 

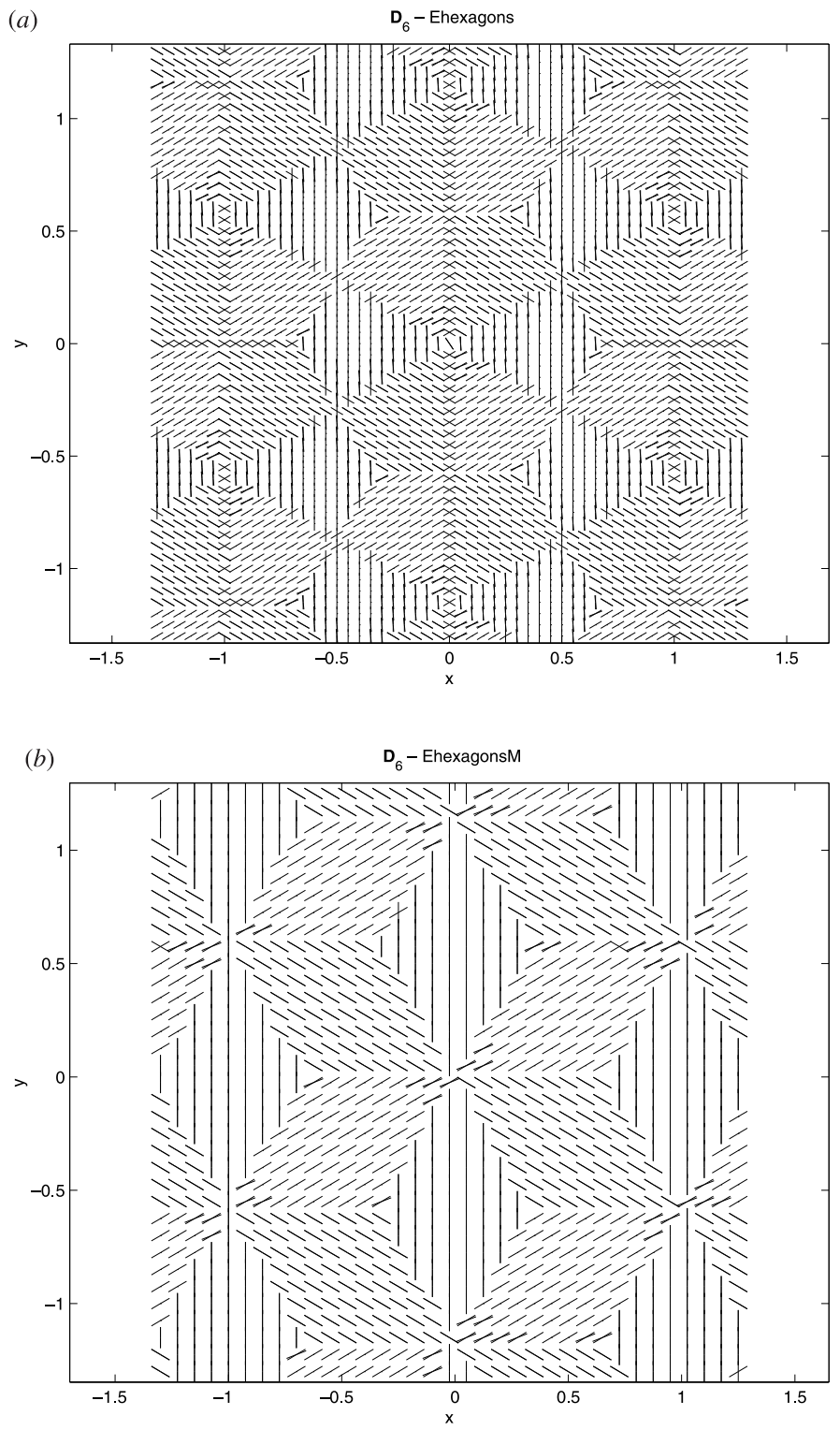

Figure 4. Hexagonal lattice: (a) EhexagonsP and (b) EhexagonsM.

Suppose that $f$ depends on a bifurcation parameter $\lambda$. Equilibria (solutions to $f=0$ ) appear in two-dimensional group orbits forced by the action of $\boldsymbol{T}^{2}$. These orbits of solutions have a unique representative of the form $\left(x_{1}, x_{2}\right) \in \mathbb{R}^{2}$ with $x_{1}, x_{2} \geqslant 0$. Therefore, we find all equilibria by solving the restricted system of equations $f: \mathbb{R}^{2} \times \mathbb{R} \rightarrow \mathbb{R}^{2}$. These equilibria are written up to conjugacy in terms of equations in $p\left(x_{1}^{2}, x_{2}^{2}, \lambda\right)$ in table 13 .

We can expand $f$ to third order by writing

$$
p\left(x_{1}^{2}, x_{2}^{2}, \lambda\right)=\lambda+A x_{1}^{2}+B x_{2}^{2}+\cdots
$$



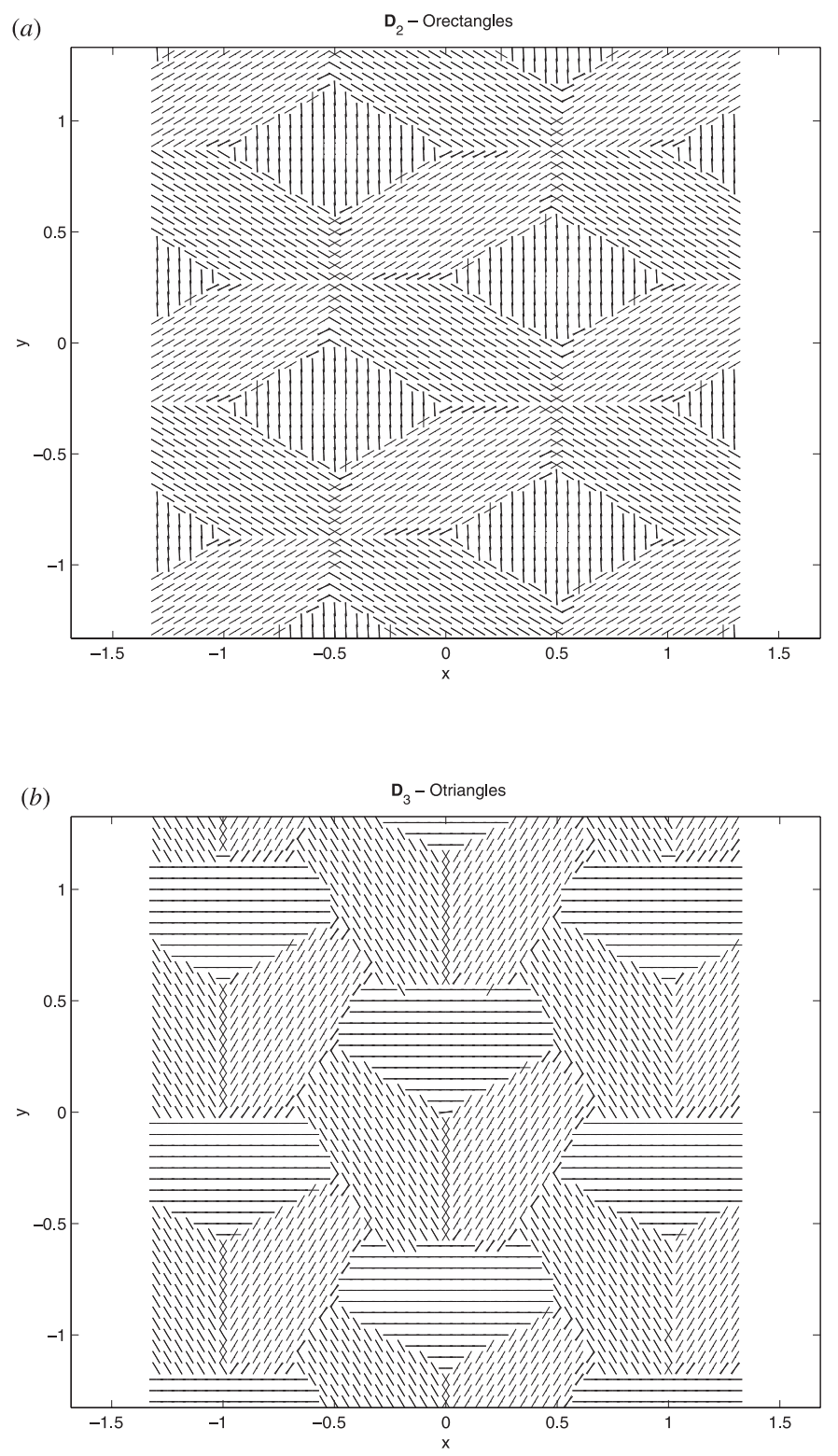

Figure 5. Hexagonal lattice: $(a)$ odd rectangles, $(b)$ odd triangles and $(c)$ odd hexagons.

where we have scaled the coefficient of $\lambda$ to be 1 . Note that when $A \neq B$, there are no submaximal solutions near the origin.

The stability of solutions is found by determining the signs of eigenvalues of the $4 \times 4$ Jacobian matrix of $f: \mathbb{C}^{2} \times \mathbb{R} \rightarrow \mathbb{C}^{2}$. The torus action forces two of these eigenvalues to be 0 ; the other two eigenvalues are obtained from the $2 \times 2$ Jacobian matrix of $f: \mathbb{R}^{2} \times \mathbb{R} \rightarrow \mathbb{R}^{2}$. 
$(c)$

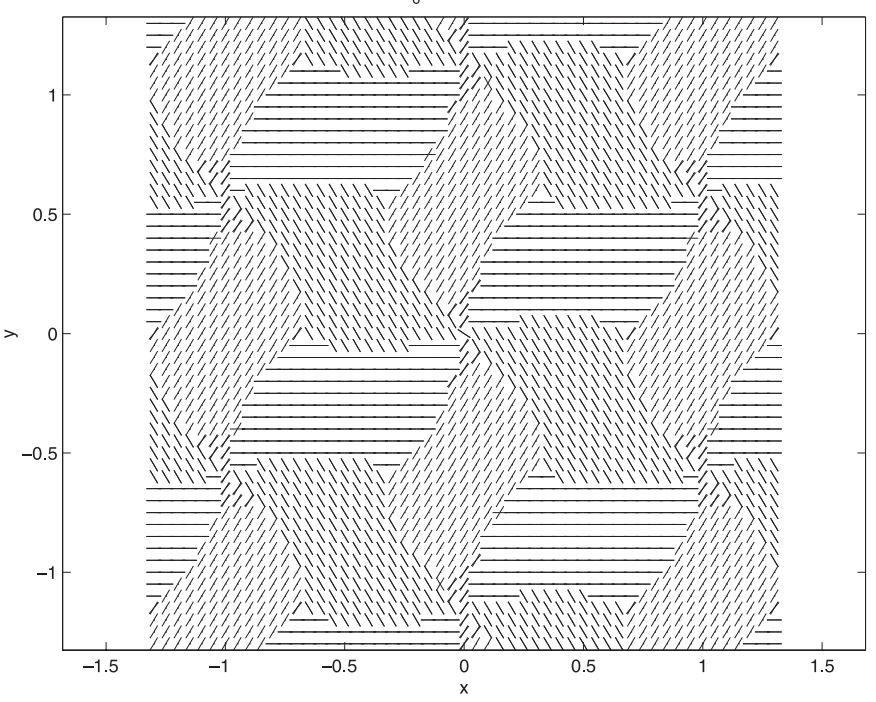

Figure 5. Continued.

Table 13. Classification up to conjugacy of centre manifold equilibria on a rhombic lattice.

\begin{tabular}{ll}
\hline Solution type & Bifurcation equations \\
\hline Trivial & $x_{1}=x_{2}=0$ \\
Rolls & $p\left(x_{1}^{2}, 0, \lambda\right)=0 ; x_{1}>0 ; x_{2}=0$ \\
Rhombs & $p\left(x_{1}^{2}, x_{1}^{2}, \lambda\right)=0 ; x_{1}=x_{2}>0$ \\
Submaximal & $p\left(x_{1}^{2}, x_{2}^{2}, \lambda\right)=0 ; p\left(x_{2}^{2}, x_{1}^{2}, \lambda\right)=0 ; 0<x_{1}<x_{2}$ \\
\hline
\end{tabular}

That Jacobian matrix is (on suppressing the explicit dependence on $\lambda$ )

$$
\left(\begin{array}{cc}
p\left(x_{1}^{2}, x_{2}^{2}\right)+2 x_{1}^{2} p_{1}\left(x_{1}^{2}, x_{2}^{2}\right) & 2 x_{1} x_{2} p_{2}\left(x_{1}^{2}, x_{2}^{2}\right) \\
2 x_{1} x_{2} p_{2}\left(x_{2}^{2}, x_{1}^{2}\right) & p\left(x_{2}^{2}, x_{1}^{2}\right)+2 x_{2}^{2} p_{1}\left(x_{2}^{2}, x_{1}^{2}\right)
\end{array}\right)
$$

where $p_{j}$ indicates differentiation of $p$ with respect to its $j$ th variable. Using the entries in table 13 we can compute these matrices in the case of trivial, roll and rhomb solutions. The results are:

$$
\begin{aligned}
& p(0,0, \lambda) I_{2} \\
& \left(\begin{array}{cc}
2 x_{1}^{2} p_{1}\left(x_{1}^{2}, 0, \lambda\right) & 0 \\
0 & p\left(0, x_{1}^{2}, \lambda\right)
\end{array}\right) \\
& 2 x_{1}^{2}\left(\begin{array}{cc}
p_{1}\left(x_{1}^{2}, x_{1}^{2}, \lambda\right) & p_{2}\left(x_{1}^{2}, x_{1}^{2}, \lambda\right) \\
p_{2}\left(x_{1}^{2}, x_{1}^{2}, \lambda\right) & p_{1}\left(x_{1}^{2}, x_{1}^{2}, \lambda\right)
\end{array}\right) .
\end{aligned}
$$

Theorem 5.2. Assume that $A \neq 0$ and $A \neq \pm B$, then near the origin the direction of branching of solutions and their stability is determined by the entries in table 14.

The theorem is proved by elementary applications of the implicit function theorem and direct calculations. Note that if the centre manifold equation depended on a second parameter 
Table 14. Stability of equilibria in generic bifurcations on a rhombic lattice.

\begin{tabular}{lll}
\hline Solution type & Direction of branching & Asymptotic stability \\
\hline Trivial & $x_{1}=x_{2}=0$ & $\lambda<0$ \\
Rolls & $\lambda=-A x_{1}^{2}$ & $A<0 ; B<A$ \\
Rhombs & $\lambda=-(A+B) x_{1}^{2}$ & $A+B<0 ; A<B$ \\
\hline
\end{tabular}

$\rho$, then for special values of $\rho$ these non-degeneracy conditions will fail, thus forcing changes in stability of the axial solutions and the existence of submaximal equilibria.

\section{The square lattice}

As with rhombic lattices, the scalar and pseudoscalar actions of $\Gamma_{\mathcal{L}}$ on $\mathbb{C}^{2}$ on the square lattice yield identical equivariants and bifurcation theories, see remark 3.2.

Lemma 5.3. The general equivariant mapping on the square lattice shortest wavevector representation has the form (5.1).

Proof. Let $f: \mathbb{C}^{2} \rightarrow \mathbb{C}^{2}$ be a square lattice equivariant. As in the proof of lemma 5.1 the torus symmetry and $\xi^{2}(z)=\bar{z}$ symmetry imply that $f$ is determined by the mapping $g: \mathbb{R}^{2} \rightarrow \mathbb{R}^{2}$ of the form

$$
g\left(x_{1}, x_{2}\right)=\left(p\left(x_{1}^{2}, x_{2}^{2}\right) x_{1}, q\left(x_{1}^{2}, x_{2}^{2}\right) x_{2}\right)
$$

where $p$ and $q$ are real-valued. The effective action of $\Gamma_{\mathcal{L}}$ on the real subspace $\left(x_{1}, x_{2}\right) \in \mathbb{R}^{2}$ is identical with the action in the rhombic case, since $\kappa$ acts trivially on $\mathbb{R}^{2}$.

It follows from lemma 5.3, and its proof, that the bifurcation theory for the square lattice is identical to the bifurcation theory for the rhombic lattice (in the shortest wavevector case) with the single exception that rhomb solutions are identified in the analysis with square solutions. Indeed, the solutions on the square lattice have different symmetries and the discussion in physical space (rather than centre manifold space) reflects these differences.

\section{The hexagonal lattice: even case}

Buzano and Golubitsky [5] made a detailed analysis of bifurcations corresponding to shortest wavevectors on the hexagonal lattice. By examining the general $\Gamma_{\mathcal{L}}$-equivariant mapping they proved that generically the only solutions that bifurcate from the trivial solution are rolls and hexagons. A theorem due to Ihrig and Golubitsky $[8,11]$ states that whenever there is a nonzero quadratic equivariant map, then generically all solutions obtained using the equivariant branching lemma are unstable. In the case of the scalar representation on the hexagonal lattice there is precisely one non-zero equivariant quadratic mapping.

As observed much earlier by Busse [4] when studying transitions in convection systems, there is a way around this pattern selection conundrum. By varying a second parameter $\rho$, it is possible to arrive at certain critical values of $\rho$ where the equivariant quadratic vanishes at the transition. Then, for small perturbations of $\rho$ away from these critical values, the branches of hexagons and rolls can undergo secondary bifurcations and become stable. For RayleighBénard convection, the determination of such a critical value of $\rho$ is straightforward, since the quadratic term vanishes for an ideal Boussinesq fluid. In that case, $\rho$ may be interpreted as a deviation of a fluid from ideal Boussinesq. In general, it may be quite difficult to find such critical values of $\rho$. 
Depending on cubic and higher-order terms, there are many bifurcation problems that are possible at critical $\rho$ values. Buzano and Golubitsky [5] used singularity theory methods to classify all of the generic bifurcations at such critical values and their universal unfoldings. They also showed precisely which cubic, fourth- and fifth-order terms need to be calculated to prove that higher-order terms in the expansion of the centre manifold vector field $f$ do not matter-in the sense that the bifurcation diagrams and stability of equilibria are qualitatively the same regardless of the exact values of coefficients in higher-order terms.

The secondary bifurcations from axial subgroups produce solutions with submaximal symmetry. In this case, triangles and rectangles are produced. The triangle solutions can be stable in certain cases, but then only depending on the sign of a fifth-order term. Triangle solution eigenfunctions have the form $\left(z_{1}, z_{1}, z_{1}\right)$ with $z_{1} \notin \mathbb{R}$. Rectangle solutions have the form $(x, y, y)$ with $x, y \in \mathbb{R} ; x \neq y ; y \neq 0$. Planforms associated with these solution types are shown in figure 6 with $z_{1}=1-\mathrm{i}$ (triangles) and $x=2 ; y=1$ (rectangles). The planforms will change with different choices of $z_{1}$ and $x, y$.

\section{The hexagonal lattice: odd case}

So far, we have shown that there are four axial subgroups: hexagons, rolls, rectangles and triangles. The complete discussion of stability and secondary bifurcations of axial solutions differs drastically from any of the other cases. We will show, for example, that the variation of one additional parameter $\rho$ can lead to a variety of secondary time periodic solutions. In the next section we show that there are no non-zero equivariant quadratics in the pseudoscalar hexagonal lattice (though there is an equivariant quartic), see theorem 6.1. For the moment, we consider the consequences of this fact.

There are two bifurcation variants in Bénard convection. In the idealized bifurcation problem an infinite fluid layer is heated from below and the transition from pure conduction to convection is modelled. The exact model depends on the boundary conditions. In the simplest cases the lateral boundary conditions are assumed to be periodic with respect to some planar lattice. The top and bottom conditions may either be different (no cover on top) or identical. When they are identical a new symmetry is introduced into the model - the midplane reflection. The midplane reflection happens to act on centre subspaces as $z \mapsto-z$. Note that the midplane reflection forces equivariant quadratics to be zero; but in a way that is different from the assumption of an ideal Boussinesq fluid.

Golubitsky et al [9] studied the scalar (even) bifurcation on the hexagonal lattice with the midplane reflection in the shortest wavevector case. They found that four solutions exist (hexagons, triangles, rectangles and rolls). Moreover, rectangles are never stable near bifurcation; rolls can be determined to be stable at third order; and at third order either triangles or hexagons can be stable — exactly which solution is stable depends on the sign of a fifth-order coefficient.

Since there are no non-zero quadratics in the hexagonal lattice odd case, the cubic truncation has $z \mapsto-z$ as a symmetry; that is, the cubic truncation is identical in form to the cubic truncation in the even hexagonal lattice with the midplane reflection. Therefore, it can be determined whether rolls can be stable at third order and whether either hexagons or triangles can be stable. For reasons that differ slightly from the midplane reflection case, rectangles are generically not stable near bifurcation, and hexagons and triangles may be stable, but that determination requires the computation of a fifth-order term. As mentioned previously, an important difference between the even and odd hexagonal lattice calculations concerns the type of secondary states obtained through $\rho$ variation. 

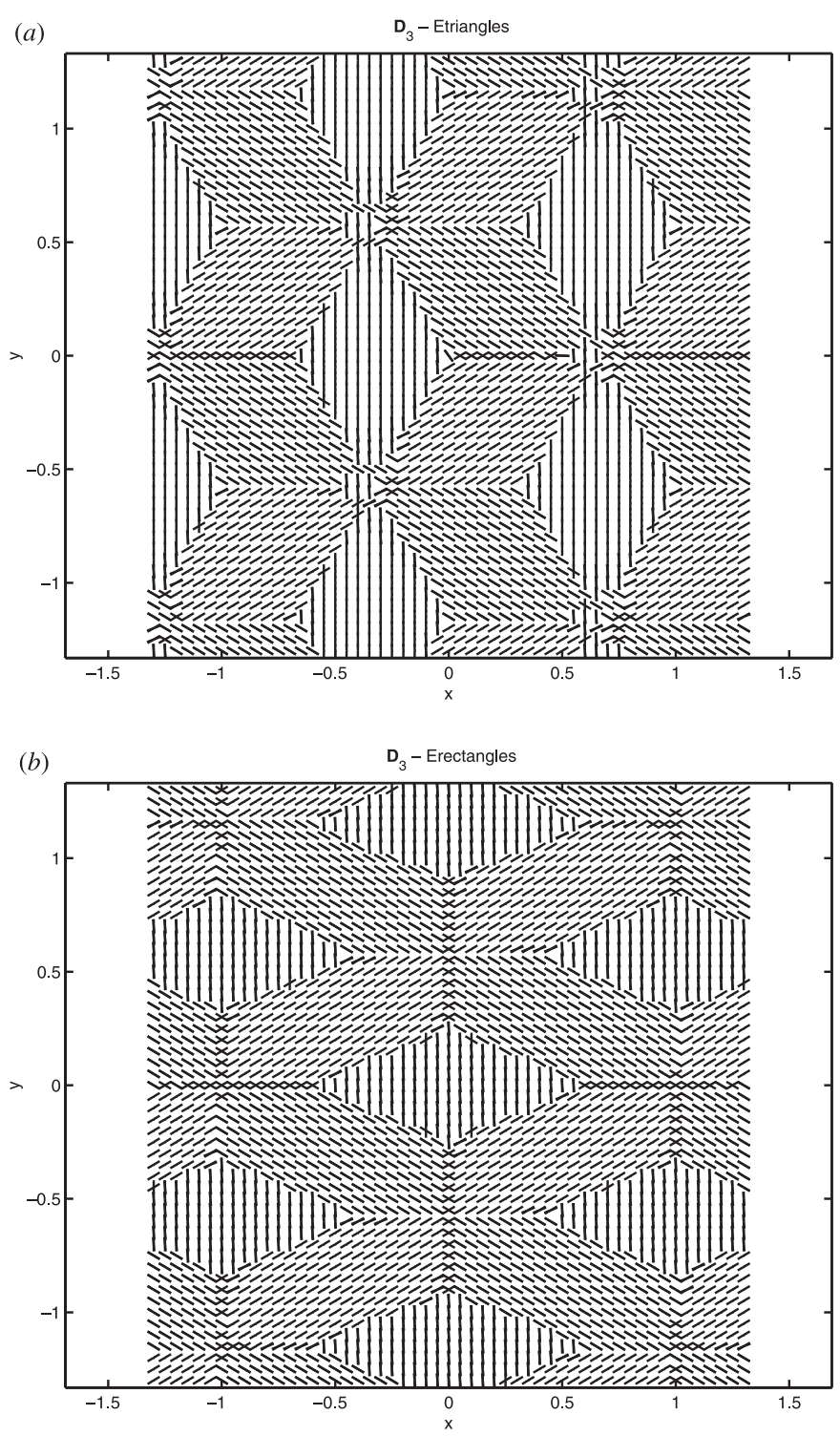

Figure 6. Hexagonal lattice: $(a)$ even triangles and $(b)$ even rectangles.

\section{Branching of axials: hexagonal odd case}

Next, we compute the direction of branching and stability of the four types of axial solutions supported on a hexagonal lattice in the odd case. These computations require knowledge of the $\Gamma_{\mathcal{L}}$-equivariant mappings on $\mathbb{C}^{3}$ up to fifth order. (Only third order is needed for the direction of branching.) We focus on the direction of branching in this section and on stability in the next.

Let $f: \mathbb{C}^{3} \rightarrow \mathbb{C}^{3}$ be $\Gamma_{\mathcal{L}}$-equivariant. Write $f$ as

$$
f(z)=\left(f_{1}(z), f_{2}(z), f_{3}(z)\right)
$$


where $f_{j}: \mathbb{C}^{3} \rightarrow \mathbb{C}$. Commutativity with respect to $\xi^{2}$ implies

$$
\begin{aligned}
& f_{2}(z)=f_{1}\left(z_{2}, z_{3}, z_{1}\right) \\
& f_{3}(z)=f_{1}\left(z_{3}, z_{1}, z_{2}\right) .
\end{aligned}
$$

Therefore,

$$
f(z)=\left(P\left(z_{1}, z_{2}, z_{3}\right), P\left(z_{2}, z_{3}, z_{1}\right), P\left(z_{3}, z_{1}, z_{2}\right)\right)
$$

where $P: \mathbb{C}^{3} \rightarrow \mathbb{C}$.

Theorem 6.1. Up to fifth order the function $P(z)$ has the form

$$
P(z)=Q(z) z_{1}+\gamma\left(\left|z_{2}\right|^{2}-\left|z_{3}\right|^{2}\right) \bar{z}_{2} \bar{z}_{3}+\eta \bar{z}_{1}\left(\bar{z}_{2} \bar{z}_{3}\right)^{2}
$$

where $Q(z)$ is the real-valued function

$$
\begin{aligned}
& Q(z)=\lambda+\alpha\left|z_{1}\right|^{2}+\beta\left(\left|z_{2}\right|^{2}+\left|z_{3}\right|^{2}\right) \\
& +\delta\left|z_{1}\right|^{4}+\epsilon\left|z_{1}\right|^{2}\left(\left|z_{2}\right|^{2}+\left|z_{3}\right|^{2}\right)+\varphi\left(\left|z_{2}\right|^{2}+\left|z_{3}\right|^{2}\right)^{2}+\psi\left(\left|z_{2}\right|^{2}-\left|z_{3}\right|^{2}\right)^{2}
\end{aligned}
$$

and all coefficients are real.

We use theorem 6.1 to compute the direction of branching of the axial planforms up to third order. Compute

$$
\begin{array}{ll}
\text { hexagons: } & \lambda=-\left((\alpha+2 \beta) x^{2}+(\delta+2 \epsilon+4 \varphi+\eta) x^{4}\right) \\
\text { triangles: } & \lambda=-\left((\alpha+2 \beta) y^{2}+(\delta+2 \epsilon+4 \varphi-\eta) y^{4}\right) \\
\text { rectangles: } & \lambda=-\left((\alpha+\beta) x^{2}+(\delta+\epsilon+\varphi+\psi) x^{4}\right) \\
\text { rolls: } & \lambda=-\left(\alpha x^{2}+\delta x^{4}\right) .
\end{array}
$$

Note that all of these branches are pitchfork bifurcations. Contrast this with the even case where branches of hexagons are generically transcritical. Assuming that the trivial homogeneous solution is stable for $\lambda<0$ and loses stability at $\lambda=0$, then axial solutions can be stable only when they are supercritical. (Then the eigenvalue of the Jacobian in the direction of the fixed-point subspace is negative or stable.)

\section{Equivariant mappings: proof of theorem 6.1}

We have used commutativity of an equivariant map $f$ with respect to $\xi^{2}$ to write $f$ in terms of the coordinate function $P$. The form of $P$ is restricted by the actions of $\xi^{3}, \kappa$ and $T^{2}$ (see table 4). These restrictions are:

$$
\begin{aligned}
& P(\bar{z})=\overline{P(z)} \\
& P\left(-z_{1},-z_{3},-z_{2}\right)=-P(z) \\
& P\left(\mathrm{e}^{\mathrm{i} \theta} z_{1}, z_{2}, \mathrm{e}^{-\mathrm{i} \theta} z_{3}\right)=\mathrm{e}^{\mathrm{i} \theta} P(z) \\
& P\left(z_{1}, \mathrm{e}^{\mathrm{i} \theta} z_{2}, \mathrm{e}^{-\mathrm{i} \theta} z_{3}\right)=P(z) .
\end{aligned}
$$

The equivariance condition (6.6) implies that

$$
P(z)=R\left(\left|z_{1}\right|^{2}, z_{2},\left|z_{3}\right|^{2}, z_{1} z_{3}, \bar{z}_{1} \bar{z}_{3}\right) z_{1}+S\left(\left|z_{1}\right|^{2}, z_{2},\left|z_{3}\right|^{2}, z_{1} z_{3}, \bar{z}_{1} \bar{z}_{3}\right) \bar{z}_{3} .
$$

This presentation of $P$ is not unique, since

$$
\left(\bar{z}_{1} \bar{z}_{3}\right) z_{1}=\left|z_{1}\right|^{2} \bar{z}_{3} \quad \text { and } \quad\left(z_{1} z_{3}\right) \bar{z}_{3}=\left|z_{3}\right|^{2} z_{1} .
$$


Using these relations we can write $P$ uniquely as

$$
P(z)=R\left(\left|z_{1}\right|^{2}, z_{2},\left|z_{3}\right|^{2}, z_{1} z_{3}\right) z_{1}+S\left(\left|z_{1}\right|^{2}, z_{2},\left|z_{3}\right|^{2}, \bar{z}_{1} \bar{z}_{3}\right) \bar{z}_{3} .
$$

The invariance condition (6.7) implies that

$$
\begin{aligned}
& R\left(\left|z_{1}\right|^{2}, \mathrm{e}^{\mathrm{i} \theta} z_{2},\left|z_{3}\right|^{2}, \mathrm{e}^{-\mathrm{i} \theta} z_{1} z_{3}\right)=R\left(\left|z_{1}\right|^{2}, z_{2},\left|z_{3}\right|^{2}, z_{1} z_{3}\right) \\
& S\left(\left|z_{1}\right|^{2}, \mathrm{e}^{\mathrm{i} \theta} z_{2},\left|z_{3}\right|^{2}, \mathrm{e}^{\mathrm{i} \theta} \bar{z}_{1} \bar{z}_{3}\right)=S\left(\left|z_{1}\right|^{2}, z_{2},\left|z_{3}\right|^{2}, \bar{z}_{1} \bar{z}_{3}\right) \mathrm{e}^{\mathrm{i} \theta} .
\end{aligned}
$$

Therefore,

$$
\begin{gathered}
R\left(\left|z_{1}\right|^{2}, z_{2},\left|z_{3}\right|^{2}, z_{1} z_{3}\right) z_{1}=T\left(\left|z_{1}\right|^{2},\left|z_{2}\right|^{2},\left|z_{3}\right|^{2}, z_{1} z_{2} z_{3}\right) z_{1} \\
S\left(\left|z_{1}\right|^{2}, z_{2},\left|z_{3}\right|^{2}, \bar{z}_{1} \bar{z}_{3}\right) \bar{z}_{3}=U\left(\left|z_{1}\right|^{2},\left|z_{2}\right|^{2},\left|z_{3}\right|^{2}, \bar{z}_{1} \bar{z}_{2} \bar{z}_{3}\right) \bar{z}_{2} \bar{z}_{3} \\
+V\left(\left|z_{1}\right|^{2},\left|z_{2}\right|^{2},\left|z_{3}\right|^{2}, z_{1} z_{2} z_{3}\right) z_{1} z_{3} \bar{z}_{3} .
\end{gathered}
$$

The function $V$ is redundant; it can be included in the $T$ term. Hence

$$
\begin{aligned}
P(z)=T\left(\left|z_{1}\right|^{2},\left|z_{2}\right|^{2}+\left|z_{3}\right|^{2},\left|z_{2}\right|^{2}-\left|z_{3}\right|^{2}, z_{1} z_{2} z_{3}\right) z_{1} \\
+U\left(\left|z_{1}\right|^{2},\left|z_{2}\right|^{2}+\left|z_{3}\right|^{2},\left|z_{2}\right|^{2}-\left|z_{3}\right|^{2}, \bar{z}_{1} \bar{z}_{2} \bar{z}_{3}\right) \bar{z}_{2} \bar{z}_{3} .
\end{aligned}
$$

Next, let $u_{1}=\left|z_{2}\right|^{2}-\left|z_{3}\right|^{2}$ and $u_{2}=z_{1} z_{2} z_{3}$ and apply (6.5) to obtain

$$
\begin{aligned}
& T\left(\left|z_{1}\right|^{2},\left|z_{2}\right|^{2}+\left|z_{3}\right|^{2},-u_{1},-u_{2}\right)=T\left(\left|z_{1}\right|^{2},\left|z_{2}\right|^{2}+\left|z_{3}\right|^{2}, u_{1}, u_{2}\right) \\
& U\left(\left|z_{1}\right|^{2},\left|z_{2}\right|^{2}+\left|z_{3}\right|^{2},-u_{1},-\overline{u_{2}}\right)=-U\left(\left|z_{1}\right|^{2},\left|z_{2}\right|^{2}+\left|z_{3}\right|^{2}, u_{1}, \overline{u_{2}}\right) .
\end{aligned}
$$

It follows that

$$
\begin{gathered}
T\left(\left|z_{1}\right|^{2},\left|z_{2}\right|^{2}+\left|z_{3}\right|^{2}, u_{1}, u_{2}\right)=V\left(\left|z_{1}\right|^{2},\left|z_{2}\right|^{2}+\left|z_{3}\right|^{2}, u_{1}^{2}, u_{1} u_{2}, u_{2}^{2}\right) \\
U\left(\left|z_{1}\right|^{2},\left|z_{2}\right|^{2}+\left|z_{3}\right|^{2}, u_{1}, \overline{u_{2}}\right)=W\left(\left|z_{1}\right|^{2},\left|z_{2}\right|^{2}+\left|z_{3}\right|^{2}, u_{1}^{2}, u_{1} \overline{u_{2}}, \overline{u_{2}}{ }^{2}\right) u_{1} \\
+X\left(\left|z_{1}\right|^{2},\left|z_{2}\right|^{2}+\left|z_{3}\right|^{2}, u_{1}^{2}, u_{1} \overline{u_{2}}, \overline{u_{2}}\right) \overline{u_{2}} .
\end{gathered}
$$

Truncating $P(z)$ at fifth order we obtain

$$
P(z)=U\left(\left|z_{1}\right|^{2},\left|z_{2}\right|^{2}+\left|z_{3}\right|^{2},\left(\left|z_{2}\right|^{2}-\left|z_{3}\right|^{2}\right)^{2}\right) z_{1}+W(0)\left(\left|z_{2}\right|^{2}-\left|z_{3}\right|^{2}\right) \bar{z}_{2} \bar{z}_{3}+X(0) \bar{z}_{1}\left(\bar{z}_{2} \bar{z}_{3}\right)^{2} .
$$

Therefore, we obtain the desired form for $P(z)$ through fifth order, but all coefficients are complex numbers. Using (6.4) we obtain the same form for $P$ but the coefficients are now all real.

\section{Stability of axial solutions}

Suppose that $x_{0}$ is an equilibrium. Then the form of the Jacobian matrix $(\mathrm{d} f)_{x_{0}}$ is restricted in two ways by symmetry. First, $(\mathrm{d} f)_{x_{0}}$ commutes with the action of the isotropy subgroup $\Sigma_{x_{0}}$ of $x_{0}$. Second, the continuous torus symmetry forces some of the eigenvalues of $(\mathrm{d} f)_{x_{0}}$ to be zero.

More precisely, commutativity forces $(\mathrm{d} f)_{x_{0}}$ to be block diagonal with respect to the isotypic components of the isotropy subgroup $\Sigma_{x_{0}}$. An isotypic component of a group $\Sigma$ is the sum of all irreducible subspaces isomorphic to a given irreducible representation. For example, $\operatorname{Fix}(\Sigma)$ is the isotypic component corresponding to the trivial representation of $\Sigma$. Every representation $V$ can be written uniquely as a direct sum of isotypic components

$$
V=\operatorname{Fix}(\Sigma) \oplus W_{1} \oplus \cdots \oplus W_{s} .
$$

Moreover, if $A: V \rightarrow V$ is linear and commutes with $\Sigma$, then $A\left(W_{j}\right) \subset W_{j}$ (see [8]). 
Table 15. The isotypic components.

\begin{tabular}{lllll}
\hline$\Sigma$ & $\operatorname{Fix}(\Sigma)$ & $W_{1}$ & $W_{2}$ & $W_{3}$ \\
\hline Ohexagons & $(1,1,1)$ & $(-2,1,1),(1,1,-2)$ & $(\mathrm{i}, \mathrm{i}, \mathrm{i})$ & $(-2 \mathrm{i}, \mathrm{i}, \mathrm{i}),(\mathrm{i}, \mathrm{i},-2 \mathrm{i})$ \\
Otriangles & $(\mathrm{i}, \mathrm{i}, \mathrm{i})$ & $z_{1}+z_{2}+z_{3}=0$ & $(1,1,1)$ & - \\
Orectangles & $(0,1,-1)$ & $(1,0,0),(0,1,1)$ & $(0, \mathrm{i},-\mathrm{i})$ & $(\mathrm{i}, 0,0),(0, \mathrm{i}, \mathrm{i})$ \\
Orolls & $(1,0,0)$ & $z_{1}=0$ & $(\mathrm{i}, 0,0)$ & - \\
\hline
\end{tabular}

Table 16. Kernels of actions on isotypic components.

\begin{tabular}{lllll}
\hline$\Sigma$ & $\operatorname{Fix}(\Sigma)$ & $W_{1}$ & $W_{2}$ & $W_{3}$ \\
\hline Ohexagons & $\mathbb{Z}_{6}(\xi)$ & $\mathbb{Z}_{2}\left(\xi^{3}\right)$ & $\mathbb{Z}_{3}\left(\xi^{2}\right)$ & $\mathbf{1}$ \\
Otriangles & $\boldsymbol{D}_{3}\left(\xi^{2}, \kappa \xi\right)$ & $\mathbf{1}$ & $\mathbb{Z}_{3}\left(\xi^{2}\right)$ & - \\
Orectangles & $\boldsymbol{D}_{2}\left(\xi^{3}, \kappa\right)$ & $\mathbb{Z}_{2}\left(\xi^{3}\right)$ & $\mathbb{Z}_{2}(\kappa)$ & $\mathbb{Z}_{2}\left(\kappa \xi^{3}\right)$ \\
Orolls & $\boldsymbol{O}(2) \times \mathbb{Z}_{4}(\rho)$ & $\mathbf{1}$ & {$\left[0, \theta_{2}\right]+\mathbb{Z}_{2}\left(\kappa\left[\frac{1}{2}, 0\right]\right)$} & - \\
\hline
\end{tabular}

There are two ways to compute the restriction forced by commutativity-explicitly and abstractly. In fact, both are useful. The abstract approach tells us the eigenvalue structure of $(\mathrm{d} f)_{x_{0}}$ whose byproduct is a classification of the secondary bifurcations that are possible. The explicit calculations (which are often simplified by the abstract calculations) allow us to compute the various combinations of solutions and their stability that are possible - the generic bifurcation diagrams. We will perform both calculations beginning with the abstract and later the specific.

The isotypic decompositions of the isotropy subgroups of the four axial solutions are listed in table 15 and the nullvectors of $(\mathrm{d} f)_{x_{0}}$ that are forced by symmetry are listed in table 18 . The eigenvalue structure of $(\mathrm{d} f)_{x_{0}}$ is recorded in table 19.

\section{Isotypic components}

The isotypic components of the four axial subgroups are given in table 15. It is straightforward to check that each subspace is invariant under the action of the relevant subgroup. Each subspace is either irreducible or the sum of two isomorphic irreducible subspaces. The spaces $W_{1} \cong \mathbb{C}^{2}$ for triangles and $W_{1} \cong \mathbb{R}^{2}$ and $W_{3} \cong \mathbb{R}^{2}$ for rectangles are sums of two isomorphic irreducible subspaces; recall that $\boldsymbol{D}_{3}$ has only one two-dimensional irreducible representation. Showing that the remaining isotypic components are irreducible is straightforward except, perhaps, the subspace $W_{1}$ for rolls. To show that this subspace is irreducible, note that the only invariant subspace that contains $(0,1,0)$ is $\mathbb{C}^{2}$, since the symmetry $\kappa\left[\frac{1}{2}, 0\right]$ maps $(0,1,0)$ to $(0,0,1)$ and the symmetry $\left[0, \frac{1}{4}\right]$ maps $(0,1,0)$ to $(0, \mathrm{i}, 0)$ and $(0,0,1)$ to $(0,0, \mathrm{i})$. To show that the irreducible representations corresponding to each isotypic component are distinct it suffices to show that the kernels of the actions of $\Sigma_{x_{0}}$ are different. The kernels of the actions on these subspaces are listed in table 16 and they are all different.

\section{Commuting linear mappings}

Let $A: \mathbb{C}^{3} \rightarrow \mathbb{C}^{3}$ be linear and commute with one of the axial subgroups $\Sigma$, such as the Jacobian matrix at an equilibrium with isotropy subgroup $\Sigma$. We know that $A$ is block diagonalized by the decomposition

$$
\mathbb{C}^{3}=\operatorname{Fix}(\Sigma) \oplus W_{1} \oplus W_{2} \quad \text { or } \quad \mathbb{C}^{3}=\operatorname{Fix}(\Sigma) \oplus W_{1} \oplus W_{2} \oplus W_{3} .
$$


Table 17. Effective actions of axial subgroups on isotypic components.

\begin{tabular}{lllll}
\hline$\Sigma$ & $\operatorname{Fix}(\Sigma)$ & $W_{1}$ & $W_{2}$ & $W_{3}$ \\
\hline Ohexagons & $\mathbf{1}$ & $\mathbb{Z}_{3}$ & $\mathbb{Z}_{2}$ & $\mathbb{Z}_{6}$ \\
Otriangles & $\mathbf{1}$ & $\boldsymbol{D}_{3}$ & $\mathbb{Z}_{2}$ & - \\
Orectangles & $\mathbf{1}$ & $\mathbb{Z}_{2}(\kappa)$ & $\mathbb{Z}_{2}\left(\xi^{3}\right)$ & $\mathbb{Z}_{2}\left(\xi^{3}\right)$ \\
Orolls & $\mathbf{1}$ & $\boldsymbol{O}(2) \times \mathbb{Z}_{4}$ & $\mathbb{Z}_{2}\left(\xi^{3}\right)$ & - \\
\hline
\end{tabular}

Table 18. The null vectors for a commuting linear map.

\begin{tabular}{llll}
\hline$\Sigma$ & $\theta_{1}$ & $\theta_{2}$ & Notes \\
\hline Ohexagons & $(\mathrm{i}, 0,-\mathrm{i})$ & $(0, \mathrm{i},-\mathrm{i})$ & \\
Otriangles & $(1,0,-1)$ & $(0,1,-1)$ & \\
Orectangles & $(0,0, \mathrm{i})$ & $(0, \mathrm{i}, \mathrm{i})$ & $(0, \mathrm{i},-\mathrm{i})=(0, \mathrm{i}, \mathrm{i})-2(0,0, \mathrm{i})$ \\
Orolls & $(\mathrm{i}, 0,0)$ & - & \\
\hline
\end{tabular}

Table 19. Eigenvalues of $A_{j}$.

\begin{tabular}{llll}
\hline$\Sigma$ & $W_{1}$ & $W_{2}$ & $W_{3}$ \\
\hline Ohexagons & $a \pm b \mathrm{i}$ & & 0 \\
& $a=\frac{1}{2} \operatorname{tr}\left(A_{1}\right)$ & & \\
Otriangles & $0, a$ (twice) & & - \\
Orectangles & No restrictions & 0 & $0, \operatorname{tr}\left(A_{3}\right)$ \\
Orolls & $a \pm \mathrm{i} b$ (twice) & 0 & - \\
\hline
\end{tabular}

Let $A_{j}=A \mid W_{j}$. There are two symmetry restrictions placed on $A_{j}: A_{j}$ commutes with the action of $\Sigma$ on $W_{j}$ and $A_{j}$ may have symmetry forced nullvectors. We consider these in turn.

First, the effective actions of $\Sigma$ on $W_{j}$ (that is, the action of $\Sigma$ modulo the kernel of the action) are listed in table 17 and the $A_{j}$ must commute with this action.

Second, the action of the continuous connected subgroup $T^{2}$ forces $A$ to have zero eigenvalues. The eigenvectors corresponding to the zero eigenvalues are given by those vectors tangent to the group action at the identity. To find these eigenvectors just compute (using table 4)

$$
\begin{aligned}
& -\left.\frac{1}{2 \pi} \frac{\mathrm{d}}{\mathrm{d} \theta_{1}}\left[\theta_{1}, 0\right] z\right|_{\theta_{1}=0}=\left(\mathrm{i} z_{1}, 0,-\mathrm{i} z_{3}\right) \\
& -\left.\frac{1}{2 \pi} \frac{\mathrm{d}}{\mathrm{d} \theta_{2}}\left[0, \theta_{2}\right] z\right|_{\theta_{2}=0}=\left(0, \mathrm{i} z_{2},-\mathrm{i} z_{3}\right) .
\end{aligned}
$$

Therefore, the nullvectors in the four cases are those listed in table 18. Note that for rectangles $(0,0, \mathrm{i})=((0, \mathrm{i}, \mathrm{i})-(0, \mathrm{i},-\mathrm{i})) / 2$. Hence $A_{2}=0$.

\section{Block decompositions}

Next we discuss the eigenvalue structure of $A_{j}$ for each of the three axial subgroups. The results are listed in table 19.

We derive the results in table 19 as follows. In the case of Ohexagons, table 18 shows that $A_{3}=0$. Table 17 shows that $A_{1}$ commutes with the action of $\mathbb{Z}_{3}$. It follows that $A_{1}$ is a multiple of a rotation matrix and that its eigenvalues are as listed.

In the case of rectangles the note in table 18 shows that $A_{2}=0$ and that the $2 \times 2$ matrix $A_{3}$ has a zero eigenvalue. Therefore, the other eigenvalue is given by the trace. There are no 
restrictions placed on $A_{1}$, since the effective action on $W_{1}$ is by the two-element group $\pm I$. Thus $A_{1}$ is just an arbitrary $2 \times 2$ matrix.

In the case of triangles, table 18 shows that two of the four eigenvalues of $A_{1}$ are zero. Since $A_{1}$ commutes with the absolutely irreducible representation of $D_{3}$ (table 17) $A_{1}$ can be written in block form with scalar multiples of the identity in each block. The zero eigenvalues force two of these blocks to be zero. Indeed, use coordinates $\operatorname{Re}\left(z_{2}\right), \operatorname{Re}\left(z_{3}\right), \operatorname{Im}\left(z_{2}\right), \operatorname{Im}\left(z_{3}\right)$ on $W_{1}$, then $A_{1}$ has the form

$$
A_{1}=\left(\begin{array}{ll}
a I_{2} & 0 \\
c I_{2} & 0
\end{array}\right)
$$

where $a, c \in \mathbb{R}$. It follows that the eigenvalues of $A_{1}$ are 0 and $a$-each repeated twice.

Finally, in the case of rolls, table 18 shows that $A_{2}=0$. Observe that table 17 shows that $A_{1}$ commutes with $\boldsymbol{O}(2) \times \mathbb{Z}_{4}$. The representation on $W_{1}$ is irreducible, but not absolute irreducible; therefore, the eigenvalues of $A_{1}$ are $a \pm \mathrm{i} b$ with each eigenvalue occurring twice.

\section{The form of $\mathrm{d} f$}

Next, we discuss the explicit form $\mathrm{d} f$. Let $z_{j}=x_{j}+\mathrm{i} y_{j}$ and $f=f_{r}+\mathrm{i} f_{i}$. Write the real coordinates in order as $x_{1}, x_{2}, x_{3}, y_{1}, y_{2}, y_{3}$. For each axial $x_{0}$ we derive the form of the $6 \times 6$ matrix $(\mathrm{d} f)_{x_{0}}$ assuming that it commutes with $\Sigma_{x_{0}}$ and has the nullvectors listed in table 18.

Hexagons $x_{0}=(x, x, x)$. The Jacobian commutes with $\xi^{3}(z)=\bar{z}$ and therefore has the block form

$$
\left(\begin{array}{ll}
B & 0 \\
0 & C
\end{array}\right)
$$

where $B$ and $C$ are $3 \times 3$ matrices. The Jacobian also commutes with $\xi^{2}\left(z_{1}, z_{2}, z_{3}\right)=$ $\left(z_{3}, z_{1}, z_{2}\right)$. Therefore, $B$ and $C$ both commute with the matrix

$$
\left(\begin{array}{lll}
0 & 0 & 1 \\
1 & 0 & 0 \\
0 & 1 & 0
\end{array}\right)
$$

which implies that $B$ and $C$ each have the form

$$
\left(\begin{array}{lll}
a & b & c \\
c & a & b \\
b & c & a
\end{array}\right) .
$$

The nullvectors listed in table 18 imply that the three columns of $C$ are identical; hence all nine entries of $C$ are equal. Therefore,

$$
(\mathrm{d} f)_{x_{0}}=\left(\begin{array}{ccc}
\left(\begin{array}{ccc}
a & b & c \\
c & a & b \\
b & c & a
\end{array}\right) & \left(\begin{array}{lll}
d & d & d \\
d & d & d \\
d & d & d
\end{array}\right)
\end{array}\right) .
$$


We now use table 15 to compute the eigenvalues of $(\mathrm{d} f)_{x_{0}}$. The fact that $(1,1,1)$ is an eigenvector of $C$ implies that $3 d$ is an eigenvalue. The fact that $(1,1,1)$ is an eigenvector of $B$ implies that $a+b+c$ is an eigenvalue. The invariant subspace $W_{1}$ has a basis $(-2,1,1),(1,1,-2)$. In this basis, $A_{1}=B \mid W_{1}$ has the form

$$
\left(\begin{array}{cc}
a-c & b-c \\
c-b & a-b
\end{array}\right)
$$

the eigenvalues of which are complex conjugates. The trace of this matrix is

$$
\operatorname{tr}\left(A_{1}\right)=2 a-b-c .
$$

Therefore, the sign of the real part of these complex conjugate eigenvalues is $2 a-b-c$ and the complex part is $\pm(b-c)$.

Rectangles $x_{0}=(0, x,-x)$. The Jacobian again commutes with $\xi^{3}$ and therefore has the block form

$$
\left(\begin{array}{ll}
B & 0 \\
0 & C
\end{array}\right)
$$

where $B$ and $C$ are $3 \times 3$ matrices. The Jacobian also commutes with $\kappa\left(z_{1}, z_{2}, z_{3}\right)=$ $\left(-z_{1},-z_{3},-z_{2}\right)$. Therefore, $B$ and $C$ both commute with the matrix

$$
\left(\begin{array}{lll}
1 & 0 & 0 \\
0 & 0 & 1 \\
0 & 1 & 0
\end{array}\right)
$$

which implies that $B$ and $C$ each have the form

$$
\left(\begin{array}{lll}
a & b & b \\
c & d & e \\
c & e & d
\end{array}\right) .
$$

The nullvectors listed in table 18 imply that

$$
C=\left(\begin{array}{lll}
f & 0 & 0 \\
g & 0 & 0 \\
g & 0 & 0
\end{array}\right)
$$

with eigenvalue $f$. Therefore,

$$
(\mathrm{d} f)_{x_{0}}=\left(\begin{array}{ccc}
\left(\begin{array}{lll}
a & b & b \\
c & d & e \\
c & e & d
\end{array}\right) & \left(\begin{array}{lll}
f & 0 & 0 \\
g & 0 & 0 \\
g & 0 & 0
\end{array}\right)
\end{array}\right) .
$$

The eigenvector $(0,1,-1)$ of $B$ has eigenvalue $d-e$. The invariant subspace $W_{1}$ has a basis $(-2,1,1),(1,1,-2)$. In this basis, $(\mathrm{d} f)_{x_{0}}$ has the form

$$
\left(\begin{array}{cc}
a & c \\
2 b & d+e
\end{array}\right)
$$

which is arbitrary. 
Rolls $x_{0}=(x, 0,0) . \quad$ The Jacobian again commutes with $\xi^{3}$ and therefore has the block form

$$
\left(\begin{array}{ll}
B & 0 \\
0 & C
\end{array}\right)
$$

where $B$ and $C$ are $3 \times 3$ matrices. The Jacobian also commutes with $\kappa\left[\frac{1}{2}, 0\right]\left(z_{1}, z_{2}, z_{3}\right)=$ $\left(z_{1},-z_{3}, z_{2}\right)$. Therefore, $B$ and $C$ both commute with the matrix

$$
\left(\begin{array}{ccc}
1 & 0 & 0 \\
0 & 0 & -1 \\
0 & 1 & 0
\end{array}\right)
$$

which implies that $B$ and $C$ each have the form

$$
\left(\begin{array}{ccc}
a & 0 & 0 \\
0 & b & c \\
0 & -c & b
\end{array}\right) .
$$

It follows that the eigenvalues of $B$ and $C$ are $a$ and $b \pm \mathrm{i} c$. The isotypic decomposition shows that the eigenvalues $b \pm \mathrm{i} c$ are identical on each block and that the eigenvalue $a$ is zero in $C$. Therefore,

$$
(\mathrm{d} f)_{x_{0}}=\left(\begin{array}{ccc}
\left(\begin{array}{ccc}
a & 0 & 0 \\
0 & b & c \\
0 & -c & b
\end{array}\right) & \left(\begin{array}{ccc}
0 & 0 & 0 \\
0 & b & c \\
0 & -c & b
\end{array}\right)
\end{array}\right) .
$$

Triangles $x_{0}=(\mathrm{i} y, \mathrm{i} y, \mathrm{i} y)$. In this case we write the Jacobian in the block form

$$
(\mathrm{d} f)_{x_{0}}=\left(\begin{array}{ll}
B & C \\
D & E
\end{array}\right)
$$

where $B, C, D, E$ are $3 \times 3$ matrices. The Jacobian commutes with $\xi^{2}\left(z_{1}, z_{2}, z_{3}\right)=\left(z_{3}, z_{1}, z_{2}\right)$. Therefore, each block commutes with the matrix

$$
\left(\begin{array}{lll}
0 & 0 & 1 \\
1 & 0 & 0 \\
0 & 1 & 0
\end{array}\right)
$$

which implies that each block has the form

$$
\left(\begin{array}{lll}
a & b & c \\
c & a & b \\
b & c & a
\end{array}\right) .
$$

The nullvectors $(1,0,-1,0,0,0)$ and $(0,1,-1,0,0,0)$ of $(\mathrm{d} f)_{x_{0}}$ imply that the three columns of $A$ are identical, as are the three columns of $C$. Thus all nine entries of $A$ are equal, as are all 
nine entries of $C$. The fact that $(1,1,1,0,0,0)$ is an eigenvector of $(\mathrm{d} f)_{x_{0}}$ (table 15) implies that $C=0$. Thus

$$
(\mathrm{d} f)_{x_{0}}=\left(\begin{array}{ccc}
\left(\begin{array}{ccc}
a & a & a \\
a & a & a \\
a & a & a
\end{array}\right) & \left(\begin{array}{lll}
b & c & d \\
d & b & c \\
c & d & b
\end{array}\right)
\end{array}\right) .
$$

Finally, $(\mathrm{d} f)_{x_{0}}$ commutes with the other generator of $\boldsymbol{D}_{3}$, namely, $\kappa \xi\left(z_{1}, z_{2}, z_{3}\right)=$ $\left(-\bar{z}_{2},-\bar{z}_{1},-\bar{z}_{3}\right)$. Therefore, $D$ commutes with

$$
\left(\begin{array}{lll}
0 & 1 & 0 \\
1 & 0 & 0 \\
0 & 0 & 1
\end{array}\right)
$$

which implies that $c=d$. Thus

$$
(\mathrm{d} f)_{x_{0}}=\left(\begin{array}{ccc}
\left(\begin{array}{ccc}
a & a & a \\
a & a & a \\
a & a & a
\end{array}\right) & \left(\begin{array}{lll}
b & c & c \\
c & b & c \\
c & c & b
\end{array}\right)
\end{array}\right) .
$$

Therefore, the eigenvalues of ( $\mathrm{d} f)_{x_{0}}$ are $3 a, 0$ (twice), $b+2 c$ and $b-c$ (twice).

Computation of eigenvalues of $(\mathrm{d} f)_{x_{0}}$

We have shown that at axial equilibria, the eigenvalues of $\mathrm{d} f$ are determined by two $3 \times 3$ matrices

$$
(\mathrm{d} f)_{x_{0}}=\left(\begin{array}{cc}
\left(\mathrm{d}_{x} f_{r}\right)_{x_{0}} & * \\
0 & \left(\mathrm{~d}_{y} f_{i}\right)_{x_{0}}
\end{array}\right) .
$$

Most choices of $W_{j}$ lead to $*=0$.

Recall (6.1) and let $P=P^{r}+\mathrm{i} P^{i}$. For a given point $\left(z_{1}, z_{2}, z_{3}\right)$ let

$$
Z_{1}=\left(z_{1}, z_{2}, z_{3}\right) \quad Z_{2}=\left(z_{2}, z_{3}, z_{1}\right) \quad Z_{3}=\left(z_{3}, z_{1}, z_{2}\right) .
$$

Then

$$
\begin{aligned}
\left(\mathrm{d}_{x} f_{r}\right) & =\left(\begin{array}{lll}
P_{x_{1}}^{r}\left(Z_{1}\right) & P_{x_{2}}^{r}\left(Z_{1}\right) & P_{x_{3}}^{r}\left(Z_{1}\right) \\
P_{x_{3}}^{r}\left(Z_{2}\right) & P_{x_{1}}^{r}\left(Z_{2}\right) & P_{x_{2}}^{r}\left(Z_{2}\right) \\
P_{x_{2}}^{r}\left(Z_{3}\right) & P_{x_{3}}^{r}\left(Z_{3}\right) & P_{x_{1}}^{r}\left(Z_{3}\right)
\end{array}\right) \\
\left(\mathrm{d}_{y} f_{i}\right) & =\left(\begin{array}{lll}
P_{y_{1}}^{i}\left(Z_{1}\right) & P_{y_{2}}^{i}\left(Z_{1}\right) & P_{y_{3}}^{i}\left(Z_{1}\right) \\
P_{y_{3}}^{i}\left(Z_{2}\right) & P_{y_{1}}^{i}\left(Z_{2}\right) & P_{y_{2}}^{i}\left(Z_{2}\right) \\
P_{y_{2}}^{i}\left(Z_{3}\right) & P_{y_{3}}^{i}\left(Z_{3}\right) & P_{y_{1}}^{i}\left(Z_{3}\right)
\end{array}\right) .
\end{aligned}
$$

Using the results of the previous subsection, we can find formulae for the eigenvalues of $(\mathrm{d} f)_{x_{0}}$. We do this for each axial in turn and summarize the results in table 20. 
Table 20. Signs of eigenvalues of $A_{j}$. Except where indicated, derivatives are evaluated at $Z_{1}$.

\begin{tabular}{lllll}
\hline$\Sigma$ & $\operatorname{Fix}(\Sigma)$ & $W_{1}$ & $W_{2}$ & $W_{3}$ \\
\hline Ohexagons & $P_{x_{1}}^{r}+P_{x_{2}}^{r}+P_{x_{3}}^{r}$ & $\frac{1}{2}\left(2 P_{x_{1}}^{r}-P_{x_{2}}^{r}-P_{x_{3}}^{r}\right) \pm \mathrm{i}\left(P_{x_{2}}^{r}-P_{x_{3}}^{r}\right)$ & $P_{y_{1}}^{i}$ & 0,0 \\
Otriangles & $P_{y_{1}}^{i}+2 P_{y_{2}}^{i}$ & $0, P_{y_{1}}^{i}-P_{y_{2}}^{i}($ twice $)$ & $P_{x_{1}}^{r}$ & - \\
Orectangles & $\left(P_{x_{1}}^{r}-P_{x_{2}}^{r}\right)\left(Z_{2}\right)$ & Equation $(7.3)$ & 0 & $0, P_{y_{1}}^{i}$ \\
Orolls & $P_{x_{1}}^{r}$ & $\left(P_{x_{1}}^{r} \pm \mathrm{i} P_{x_{2}}^{r}\right)\left(Z_{2}\right)$ (twice) & 0 & - \\
\hline
\end{tabular}

Hexagons. Using (7.1) and (7.6) we see that the eigenvalues of $\mathrm{d} f$ are determined by the four coefficients:

$$
a=P_{x_{1}}^{r}\left(Z_{1}\right) \quad b=P_{x_{2}}^{r}\left(Z_{1}\right) \quad c=P_{x_{3}}^{r}\left(Z_{1}\right) \quad d=P_{y_{1}}^{i}\left(Z_{1}\right)
$$

where $Z_{1}=(x, x, x)$. The non-zero eigenvalues are $3 d, a+b+c$ and a complex conjugate pair whose real part is $\frac{1}{2}(2 a-b-c)$ and imaginary part is $b-c$. A short calculation verifies the entries in table 20.

Rectangles. Using (7.2) and (7.6) we see that the eigenvalues of $\mathrm{d} f$ are determined by the six coefficients:

$$
\begin{array}{lll}
a=P_{x_{1}}^{r}\left(Z_{1}\right) & b=P_{x_{2}}^{r}\left(Z_{1}\right) & c=P_{x_{3}}^{r}\left(Z_{2}\right) \\
d=P_{x_{1}}^{r}\left(Z_{2}\right) & e=P_{x_{2}}^{r}\left(Z_{2}\right) & f=P_{y_{1}}^{i}\left(Z_{1}\right)
\end{array}
$$

where $Z_{1}=(0, x,-x)$ and $Z_{2}=(x,-x, 0)$. The non-zero eigenvalues are $f, d-e$ and the eigenvalues of the $2 \times 2$ matrix (7.3).

Rolls. Using (7.4) and (7.6) we see that the eigenvalues of $\mathrm{d} f$ are determined by the three coefficients:

$$
a=P_{x_{1}}^{r}\left(Z_{1}\right) \quad b=P_{x_{1}}^{r}\left(Z_{2}\right) \quad c=P_{x_{2}}^{r}\left(Z_{2}\right)
$$

where $Z_{1}=(x, 0,0)$ and $Z_{2}=(0,0, x)$. The non-zero eigenvalues are $a$ and a complex conjugate pair $b \pm \mathrm{i} c$.

Triangles. Using (7.5) and (7.6) we see that the eigenvalues of $\mathrm{d} f$ are determined by the three coefficients

$$
a=P_{x_{1}}^{r}\left(Z_{1}\right) \quad b=P_{y_{1}}^{i}\left(Z_{1}\right) \quad c=P_{y_{2}}^{i}\left(Z_{1}\right)
$$

where $Z_{1}=(\mathrm{i} y, \mathrm{i} y, \mathrm{i} y)$. The non-zero eigenvalues are $3 a, b+2 c$ and $b-c$ (twice).

\section{The bifurcation diagrams}

Recall from theorem 6.1 that

$$
P(z)=Q(z) z_{1}+\gamma\left(\left|z_{2}\right|^{2}-\left|z_{3}\right|^{2}\right) \bar{z}_{2} \bar{z}_{3}+\eta \bar{z}_{1}\left(\bar{z}_{2} \bar{z}_{3}\right)^{2}
$$

where

$$
\begin{aligned}
& Q(z)=\lambda+\alpha\left|z_{1}\right|^{2}+\beta\left(\left|z_{2}\right|^{2}+\left|z_{3}\right|^{2}\right) \\
& \quad+\delta\left|z_{1}\right|^{4}+\epsilon\left|z_{1}\right|^{2}\left(\left|z_{2}\right|^{2}+\left|z_{3}\right|^{2}\right)+\varphi\left(\left|z_{2}\right|^{2}+\left|z_{3}\right|^{2}\right)^{2}+\psi\left(\left|z_{2}\right|^{2}-\left|z_{3}\right|^{2}\right)^{2}
\end{aligned}
$$


Table 21. Signs of eigenvalues of $A_{j}$ near bifurcation.

\begin{tabular}{lllcc}
\hline$\Sigma$ & $\operatorname{Fix}(\Sigma)$ & $W_{1}$ & $W_{2}$ & $W_{3}$ \\
\hline Ohexagons & $\alpha+2 \beta$ & $(\alpha-\beta) \pm 2 \gamma \mathrm{i} x^{2}$ & $-\eta$ & 0,0 \\
Otriangles & $\alpha+2 \beta$ & $(\alpha-\beta), 0$ (twice) & $\eta$ & - \\
Orectangles & $\alpha+\beta$ & $\begin{array}{l}\operatorname{det}\left(A_{1}\right)=\eta(\alpha-\beta)-2 \gamma^{2} \\
\operatorname{tr}\left(A_{1}\right)=\alpha-\beta\end{array}$ & 0 & $-\eta, 0$ \\
Orolls & $\alpha$ & $(\beta-\alpha) \pm \gamma$ ix (twice) & 0 & - \\
\hline
\end{tabular}

is real-valued. Therefore,

$$
\begin{aligned}
& P^{r}(z)=Q(z) x_{1}+\gamma\left(\left|z_{2}\right|^{2}-\left|z_{3}\right|^{2}\right)\left(x_{2} x_{3}-y_{2} y_{3}\right)+\eta \operatorname{Re}\left(\bar{z}_{1}\left(\bar{z}_{2} \bar{z}_{3}\right)^{2}\right) \\
& P^{i}(z)=Q(z) y_{1}-\gamma\left(\left|z_{2}\right|^{2}-\left|z_{3}\right|^{2}\right)\left(x_{2} y_{3}+x_{3} y_{2}\right)+\eta \operatorname{Im}\left(\bar{z}_{1}\left(\bar{z}_{2} \bar{z}_{3}\right)^{2}\right) .
\end{aligned}
$$

We now compute the eigenvalues for each axial—through fifth order.

Hexagons $(x, x, x)$

$$
\begin{array}{ll}
a=2 \alpha x^{2}+4(\delta+\epsilon) x^{4} & b=2 \beta x^{2}+(2 \epsilon+8 \varphi+2 \gamma+2 \eta) x^{4} \\
d=-2 \eta x^{4} & c=2 \beta x^{2}+(2 \epsilon+8 \varphi-2 \gamma+2 \eta) x^{4} .
\end{array}
$$

The signs of the eigenvalues near bifurcation are determined by the entries in table 21 .

Rectangles $(0, x,-x)$

$$
\begin{array}{lll}
a=\eta x^{4} & d=2 \alpha x^{2}+(4 \delta+2 \epsilon) x^{4} & c=\gamma x^{3} \\
b=2 \gamma x^{3} & e=-2 \beta x^{2}-(2 \epsilon-4 \varphi-4 \psi) x^{4} & f=-\eta x^{4} .
\end{array}
$$

The matrix (7.3) is

$$
\left(\begin{array}{cc}
a & c \\
2 b & d+e
\end{array}\right)=x^{2}\left(\begin{array}{cc}
\eta x^{2} & \gamma x \\
4 \gamma x & 2(\alpha-\beta)+4(\delta-\varphi-\psi) x^{2}
\end{array}\right) .
$$

Near the origin

$$
\operatorname{sgn}(\operatorname{det}(7.7))=\eta(\alpha-\beta)-2 \gamma^{2} \quad \text { and } \quad \operatorname{sgn}(\operatorname{tr}(7.7))=\alpha-\beta .
$$

For rectangles to be stable we need $\eta>0$ and $\operatorname{det}(7.7)>0$. Hence, we need $\alpha-\beta>0$. However, that implies $\operatorname{tr}(7.7)>0$ - and rectangles are unstable near bifurcation.

Rolls $(x, 0,0)$

$$
a=2 \alpha x^{2}+4 \delta x^{4} \quad b=(\beta-\alpha) x^{2}+(\varphi+\psi-\delta) x^{4} \quad c=-\gamma x^{3} .
$$

The signs of the eigenvalues near bifurcation are determined by the entries in table 21 . 
Triangles (iy, i $y, \mathrm{i} y)$

$$
a=\eta y^{4} \quad b=2 \alpha y^{2}+(4 \delta+4 \epsilon) y^{4} \quad c=2 \beta y^{2}+(2 \epsilon+8 \varphi+2 \eta) y^{4} .
$$

The signs of the eigenvalues near bifurcation are determined by the entries in table 21 .

These calculations may be summarized as follows:

Theorem 7.1. Suppose that the third-order coefficients $\alpha$ and $\beta$ satisfy the non-degeneracy conditions

$$
\alpha \neq \pm \beta \quad \alpha \neq 0 \quad \alpha \neq-2 \beta
$$

and the fourth- and fifth-order coefficients satisfy

$$
2 \gamma^{2} \neq \eta(\alpha-\beta) \quad \text { and } \quad \eta \neq 0 .
$$

Then near the origin the direction of branching and the stability of the four branches of axial solutions are determined by the data in table 21 and (6.3). Note that generically rectangles are never stable near codimension-zero bifurcations.

\section{Secondary bifurcations}

Secondary bifurcations occur when eigenvalues of the Jacobian cross the imaginary axis. Such eigenvalue crossings can happen even when the direction of branching does not change. For example, when $\alpha=\beta<0$ both rolls and hexagons can undergo Hopf bifurcations as long as the fourth-order coefficient $\gamma \neq 0$.

\section{Secondary Hopf bifurcations}

The roll bifurcation is an $\boldsymbol{O}(2)$ symmetry breaking Hopf bifurcation (with two complex conjugate pairs of eigenvalues crossing the imaginary axis) which (typically) generates two branches of solutions: a rotating wave and a standing wave [8]. The calculations to verify the non-degeneracy assumptions on this Hopf bifurcation are complicated and we have not carried them out. Assuming that they can be satisfied, the rotating wave will move along the direction of symmetry of the roll solution while the standing wave will have a fixed symmetry axis for all time.

The Hopf bifurcation from hexagons is caused by simple eigenvalues crossing the imaginary axis and thus yields (generically) a single branch of time periodic solutions. Again, we have not attempted to verify the non-degeneracy conditions. From tables 16 and 17 we see (when referring to the $W_{1}$ subspace) that this periodic solution will be a discrete rotating wave with two distinct symmetries: spatial rotation by $2 \pi / 3$ produces the same solution a third of a period later and rotation by $\pi$ is a symmetry for all time $t$.

\section{Secondary steady-state bifurcations}

Secondary steady-state bifurcations occur off hexagons, triangles and rectangles when $\eta \approx 0$. Another steady-state bifurcation can arise from triangles when $\alpha \approx \beta$. This bifurcation is a symmetry-breaking $\boldsymbol{D}_{3}$ symmetric steady-state bifurcation. As discussed in [8] such bifurcations (typically) produce an unstable transcritical branch of equilibria (generically there is an equivariant quadratic in this bifurcation) having a reflectional symmetry $\kappa \xi$. The normalizer of this reflection includes the one-parameter family of translations along the line of reflection (which is the line tilted at $-\pi / 6$ ). It follows from the general theory outlined next that this new solution is only a relative equilibrium and, in fact, it drifts in the direction indicated. As mentioned, this particular solution is unstable at bifurcation-but could regain stability if the branch of solutions undergoes a saddle node bifurcation. 
A review of drifting solutions

The theory of bifurcation from group orbits of equilibria was developed by Field [7] and Krupa [12]. There are three main theorems.

(a) The dynamics near a critical group orbit breaks up into a flow normal to the critical group orbit and one that is tangent to nearby group orbits.

(b) Bifurcation is controlled by the normal vector field and that vector field is $\Sigma$ equivariant. Generically, bifurcations in the normal directions are standard $\Sigma$-equivariant bifurcations.

(c) The complete bifurcation from the critical group orbit is a combination of the bifurcating state in the normal direction and drift along group orbits. Let $\Sigma_{0}$ be the isotropy subgroup of points on the bifurcated solution in the normal direction and let $N_{\Gamma}\left(\Sigma_{0}\right)$ be the normalizer of $\Sigma_{0}$. Then generically, the drift is on a $k$-torus where

$$
k=\operatorname{rank}\left(N_{\Gamma}\left(\Sigma_{0}\right) / \Sigma_{0}\right) .
$$

If $k=0$, then there is no drift.

If $k=1$, then there is drift along a circle.

If $k=2$, then there is quasiperiodic drift on a 2 -torus.

It follows from this theory that generically the $D_{3}$ symmetry-breaking secondary bifurcation from triangles described in the previous subsection will be a drifting solution with $k=1$ since in this case the normalizer $N_{\Gamma}\left(\Sigma_{0}\right)$ contains all translations along the line tilted at $-\pi / 6$ while $\Sigma_{0}=\mathbb{Z}_{2}(\kappa \xi)$ itself is finite.

\section{Acknowledgments}

We are grateful to Ian Melbourne and Ian Stewart for a number of helpful discussions concerning the structure of pseudoscalar bifurcations. This work was supported in part by grant no 96-24 from the James S McDonnell Foundation to JDC, by NSF Grant DMS-0071735 to MG and by a grant from the Leverhulme Trust to PCB. PCB wishes to thank the Mathematics Department, University of Chicago for its hospitality and support; and MG wishes to thank the Center for Biodynamics, Boston University for its hospitality and support. PJT was supported in part by an NIH training grant at the University of Chicago and in part by the Sloan-Swartz Center for Theoretical Neurobiology at the Salk Institute for Biological Studies.

\section{References}

[1] Bosch Vivancos I, Chossat P and Melbourne I 1995 New planforms in systems of partial differential equations with Euclidean symmetry Arch. Rational Mech. Anal. 131 199-224

[2] Bressloff P C, Cowan J D, Golubitsky M, Thomas P J and Wiener M C 2001 Geometric visual hallucinations, Euclidean symmetry and the functional architecture of striate cortex Phil. Trans. R. Soc. B 356 299-330

[3] Bressloff P C, Cowan J D, Golubitsky M, Thomas P J and Wiener M C 2001 What geometric visual hallucinations tell us about the visual cortex Neural Comput. to appear

[4] Busse F H 1962 Das Stabilitätsverhalten der Zellarkonvektion bei endlicher Amplitude PhD Thesis Munich University

[5] Buzano E and Golubitsky M 1983 Bifurcation involving the hexagonal lattice and the planar Bénard problem Phil. Trans. R. Soc. A $\mathbf{3 0 8} 617-67$

[6] Ermentrout G B and Cowan J D 1979 A mathematical theory of visual hallucination patterns Biol. Cybern. 34 $137-50$

[7] Field M J 1980 Equivariant dynamical systems Trans. Am. Math. Soc. 229 185-205

[8] Golubitsky M, Stewart I N and Schaeffer D G 1988 Singularities and Groups in Bifurcation Theory II (Applied Mathematics Sciences 69) (New York: Springer) 
[9] Golubitsky M, Swift J W and Knobloch E 1984 Symmetries and pattern selection in Rayleigh-Bénard convection Physica D 10 249-76

[10] Hubel D H and Wiesel T N 1974 Sequence regularity and geometry of orientation columns in the monkey striate cortex J. Comput. Neurol. 158 267-94

Hubel D H and Wiesel T N 1974 Uniformity of monkey striate cortex: a parallel relationship between field size, scatter and magnification factor J. Comput. Neurol. 158 295-306

Hubel D H and Wiesel T N 1974 Ordered arrangement of orientation columns in monkeys lacking visual experience J. Comput. Neurol. 158 307-18

[11] Ihrig E and Golubitsky M 1984 Pattern selection with $O$ (3) symmetry Physica D 13 1-33

[12] Krupa M 1990 Bifurcations from relative equilibria SIAM. J. Math. Anal. 21 1453-86

[13] Wilson H R and Cowan J 1972 Excitatory D and inhibitory interactions in localized populations of model neurons Biophys. J. 12 1-24 